\title{
Mineralocorticoid receptor antagonism limits experimental choroidal neovascularization and structural changes associated with neovascular age-related macular degeneration
}

\author{
Min Zhao 1,2,3, Irmela Mantel @ ${ }^{4}$, Emmanuelle Gelize ${ }^{1,2,3}$, Xinxin Li ${ }^{1,2,3}$, Xiaoyue Xie ${ }^{1,2,3}$, Alejandro Arboleda ${ }^{1,2,3,5}$, \\ Marie Seminel1,2,3, Rinath Levy-Boukris 1,2,3, Marilyn Dernigoghossian 1,2,3, Andrea Prunotto6, \\ Charlotte Andrieu-Soler ${ }^{7}$, Carlo Rivolta (1) 6,8, Jérémie Canonica ${ }^{4}$, Marie-Christine Naud ${ }^{1,2,3}$, \\ Sebastian Lechner $2,3,9$, Nicolette Farman ${ }^{2,3,9}$, Irene Bravo-Osuna 10,11,12, Rocio Herrero-Vanrell ${ }^{10,11,12,}$ \\ Frederic Jaisser ${ }^{2,3,9}$ \& Francine Behar-Cohen 1,2,3,13
}

Choroidal neovascularization (CNV) is a major cause of visual impairment in patients suffering from wet age-related macular degeneration (AMD), particularly when refractory to intraocular anti-VEGF injections. Here we report that treatment with the oral mineralocorticoid receptor (MR) antagonist spironolactone reduces signs of CNV in patients refractory to anti-VEGF treatment. In animal models of wet $A M D$, pharmacological inhibition of the MR pathway or endothelial-specific deletion of MR inhibits CNV through VEGFindependent mechanisms, in part through upregulation of the extracellular matrix protein decorin. Intravitreal injections of spironolactone-loaded microspheres and systemic delivery lead to similar reductions in CNV. Together, our work suggests MR inhibition as a novel therapeutic option for wet AMD patients unresponsive to anti-VEGF drugs.

\footnotetext{
${ }^{1}$ Inserm UMR_S 1138, Team 17, Centre de Recherche des Cordeliers, 75006 Paris, France. ${ }^{2}$ Sorbonne University, University of Pierre et Marie Curie, UMR_S 1138, Centre de Recherche des Cordeliers, 75006 Paris, France. ${ }^{3}$ Paris Descartes University, Sorbonne Paris Cité, UMR_S 1138, Centre de Recherche des Cordeliers, 75006 Paris, France. ${ }^{4}$ Department of Ophthalmology, University of Lausanne, Jules Gonin Eye Hospital, Fondation Asile des Aveugles, 1004 Lausanne, Switzerland. ${ }^{5}$ Ophthalmic Biophysics Center, Department of Ophthalmology, Bascom Palmer Eye Institute, University of Miami Miller School of Medicine, Miami, FL 33136, USA. ${ }^{6}$ Department of Computational Biology, Unit of Medical Genetics, University of Lausanne, 1011 Lausanne, Switzerland. ${ }^{7}$ IGMM, CNRS, Univ. Montpellier, 34293 Montpellier Cedex 5, France. ${ }^{8}$ Department of Genetics and Genome Biology, University of Leicester, Leicester LE1 7RH, UK. ${ }^{9}$ Inserm UMR_S 1138, Team 1, Centre de Recherche des Cordeliers, 75006 Paris, France. ${ }^{10}$ Department of Pharmacy and Pharmaceutical Technology, Universidad Complutense de Madrid, 28040 Madrid, Spain. ${ }^{11}$ Instituto Universitario de Farmacia Industrial, Faculty of Pharmacy, Universidad Complutense de Madrid, 28040 Madrid, Spain. ${ }^{12}$ Fundación para la Investigación-HCSC, Instituto de Investigación Sanitaria San Carlos (IdISSC), 28040 Madrid, Spain. ${ }^{13}$ Assistance Publique - Hôpitaux de Paris, Hôtel-Dieu de Paris, 75004 Paris, France. These authors contributed equally: Frederic Jaisser, Francine Behar-Cohen. Correspondence and requests for materials should be addressed to F.B.-C. (email: Francine.behar@gmail.com)
} 
A ge-related macular degeneration (AMD) is the most frequent cause of blindness in the elderly population in industrialized countries. With a global prevalence of $8 \%$, the projected number of individuals affected in 2020 is 196 million, increasing to 288 million in $2040^{1}$. Almost a third of early AMD progresses to neovascular AMD (nAMD). Choroidal neovascularization $(\mathrm{CNV})$, in which new vessels growing from the choroid toward the neuroretina underneath the macula, causes macular edema, bleeding, photoreceptors damages, and eventually end stage fibrotic scare (Supplementary Fig. 1). Aging, heredity, diet, smoking, obesity, and vascular diseases are involved in the pathogenesis of $\mathrm{AMD}^{2}$; however, the exact mechanisms leading to $\mathrm{CNV}$ remain incompletely understood.

$\mathrm{CNV}$ is not specific to AMD, it may complicate multiple other diseases affecting the retinal pigment epithelium (RPE) and the choroid, including high myopia and central serous chorioretinopathy (CSCR). The pathogenesis of CNV is complex and multifactorial. Choroidal vessels ensure nutritional and oxygen supply to the avascular outer retina containing the highly energy demanding photoreceptor cells. Choriocapillary loss, observed in nAMD eyes, may cause hypoxia and angiogenesis. A growing body of evidence also indicates that low-grade inflammation, activation of the inflammasome $e^{3,4}$, and alternative complement pathway activation play key roles in the pathogenesis of nAMD ${ }^{5}$. In addition to vascular endothelial growth factor (VEGF) family members and their receptors ${ }^{6}$, complement components and proinflammatory molecules accumulating in the RPE-choroid complex $^{7}$, such as cytokines and angiopoietins ${ }^{8}$, contribute to CNV growth. The inflammation- and hypoxia-induced downregulation of anti-angiogenic factors such as pigment epithelium-derived factor (PEDF), endostatin, and thrombospondin-1 (TSP-1) ${ }^{9}$ favors a pro-angiogenic microenvironment.

Although multiple molecular pathways have been implicated in the formation and maintenance of CNV, the treatment of nAMD currently relies on biologic compounds that only neutralize VEGF, placental growth factor (PlGF), or both without directly target inflammation ${ }^{10,11}$. Anti-VEGF drugs have strongly improved the visual prognosis of nAMD, allowing the maintenance, and even the restoration of macular function and morphology at the price of multiple intraocular injections ${ }^{12}$. However, anti-VEGFs do not allow CNV regression in nAMD ${ }^{13,14}$. In addition, they have no effect on the fibrotic scarring and may compromise long-term choroid and retinal viability 15,16 . Instead, anti-VEGF agents regulate vascular permeability, as manifested by signs such as edema, which is used to monitor the need for reinjection ${ }^{17}$. In $>40 \%$ of nAMD cases treated with intensive anti-VEGF treatment for a year, the macula remains wet, suggesting that other pathways are likely to be involved $^{18}$.

Intraocular corticosteroids, a family of potent antiinflammatory and vasoconstrictor drugs, efficiently reduce macular edema of various origins (diabetic retinopathy, retinal vein occlusion, intraocular inflammation) ${ }^{19-21}$, but show poor efficacy in nAMD. Corticosteroids bind to the glucocorticoid (GR) and mineralocorticoid receptors (MR), both expressed in the retina and choroid ${ }^{22}$. We have previously shown that experimental MR activation mimics CSCR, a retinal disease induced and aggravated by glucocorticoids and associated with subretinal fluid accumulation and frequently complicated by $\mathrm{CNV}^{23,24}$. MR antagonists (MRAs) have been shown to be efficient in $\mathrm{CSCR}^{25,26}$. In the vasculature, the MR expressed in endothelial and smooth muscle cells contributes to hypertension, vascular inflammation, and fibrosis, for which MRA have beneficial effects ${ }^{27}$. Glucocorticoids are angiostatic ${ }^{28}$, whereas mineralocorticoids have shown both pro- or anti-angiogenic effects depending on the experimental model ${ }^{29,30}$. MRAs showed various anti-angiogenic effects ${ }^{31}$, and spironolactone protected against retinal neovascularization in experimental oxygeninduced retinopathy ${ }^{32}$.

Although a growing body of evidence identifies MR as a player in vascular inflammation, fibrosis, and angiogenesis, their role in the pathogenesis of CNV has not been investigated. In this study, we show that spironolactone, an oral MRA may reduce signs of CNV activity in nAMD patients with resistant active CNV despite monthly intraocular anti-VEGF injections. Using both pharmacologic and transgenic approaches in rodents, we show that antagonism of the mineralocorticoid pathway prevents $\mathrm{CNV}$ through a VEGF-independent mechanism. We find that the benefit of spironolactone is additive with anti-VEGF therapy and mediated by the regulation of decorin. These pre-clinical and clinical results identify the $\mathrm{MR}$ as a molecular regulatory target for wet AMD.

\section{Results}

Spironolactone reduces $\mathrm{CNV}$ activity in refractory nAMD. Twenty patients with nAMD presenting with refractory intra- or subretinal fluid despite monthly intravitreal injections of antiVEGF ( $\geq 12$ months anti-VEGF treatment, $\geq 6$ months refractoriness despite monthly injections, using the same anti-VEGF molecule (Aflibercept in 13 eyes/Ranibizumab in 8 eyes), $\geq 350 \mu \mathrm{m}$ on thickest A-scan on optical coherence tomography (OCT)) consented to participate to a prospective pilot study. Refractoriness was defined as no reduction in exudative signs during the last 6 months. In addition to monthly injections of anti-VEGF during the 6-months study period, continuing with the same anti-VEGF molecule as used during the $\geq 6$ months before inclusion, they were prescribed adjuvant oral spironolactone $(25 \mathrm{mg} /$ day week $1,50 \mathrm{mg}$ /day until Month 3, $25 \mathrm{mg} /$ day until Month 4, $0 \mathrm{mg}$ until Month 6). In 21 eyes of 20 patients with refractory nAMD (13 females, mean age $76.3 \pm 7.7$ (SD) years received $37.2 \pm 17.1$ (mean \pm SD) anti-VEGF injections given over a mean period of $46.0 \pm 19.8$ (SD) months prior to study enrollment), statistically significant changes in structural outcome measures, maximal at Month 3 compared to baseline, were observed on spectral domain OCT (SD-OCT) at various time points during spironolactone treatment (Fig. 1). The effect was seen in central retinal thickness $(p=0.011)$ and volume $(p=$ $0.012)$, in the foveal thickness $(p=0.039)$, the thickest cystic changes $(p=0.040)$, and subretinal fluid $(p=0.014)$, which all quantify macular edema (Table 1 and Fig. 1 , using paired Wilcoxon signed-rank tests). The improvements observed on SDOCT were lost after stopping the spironolactone at Month 4 although anti-VEGF injections alone were continued (Table 1 and Fig. 1). Individual treatment responses are imaged by thickness maps and a selected B-scan OCT for baseline, 3 and 6 months (horizontal line) for all eyes, obtained with the follow-up mode of the Spectralis OCT (Heidelberg Engineering, Germany) (Supplementary Figs. 2-4). There was a high heterogeneity in the patient's response to the treatment, with about $50 \%$ showing a reduction of fluid at 3 months that could be attributable to spironolactone treatment, which without a control groups remains to be confirmed by a randomized controlled study. Adjuvant oral spironolactone therapy was subjectively well tolerated by most patients. However, two (10\%) of them discontinued the drug after 2 months due to increased plasma potassium levels. On the basis of an intention-to-treat approach, these patients were maintained in the analysis.

Anti-angiogenic effects of MRA in a rat nAMD model. The above clinical results of anti-edematous effect of MRA might either be linked to an anti-angiogenic effect on $\mathrm{CNV}$ or be an 

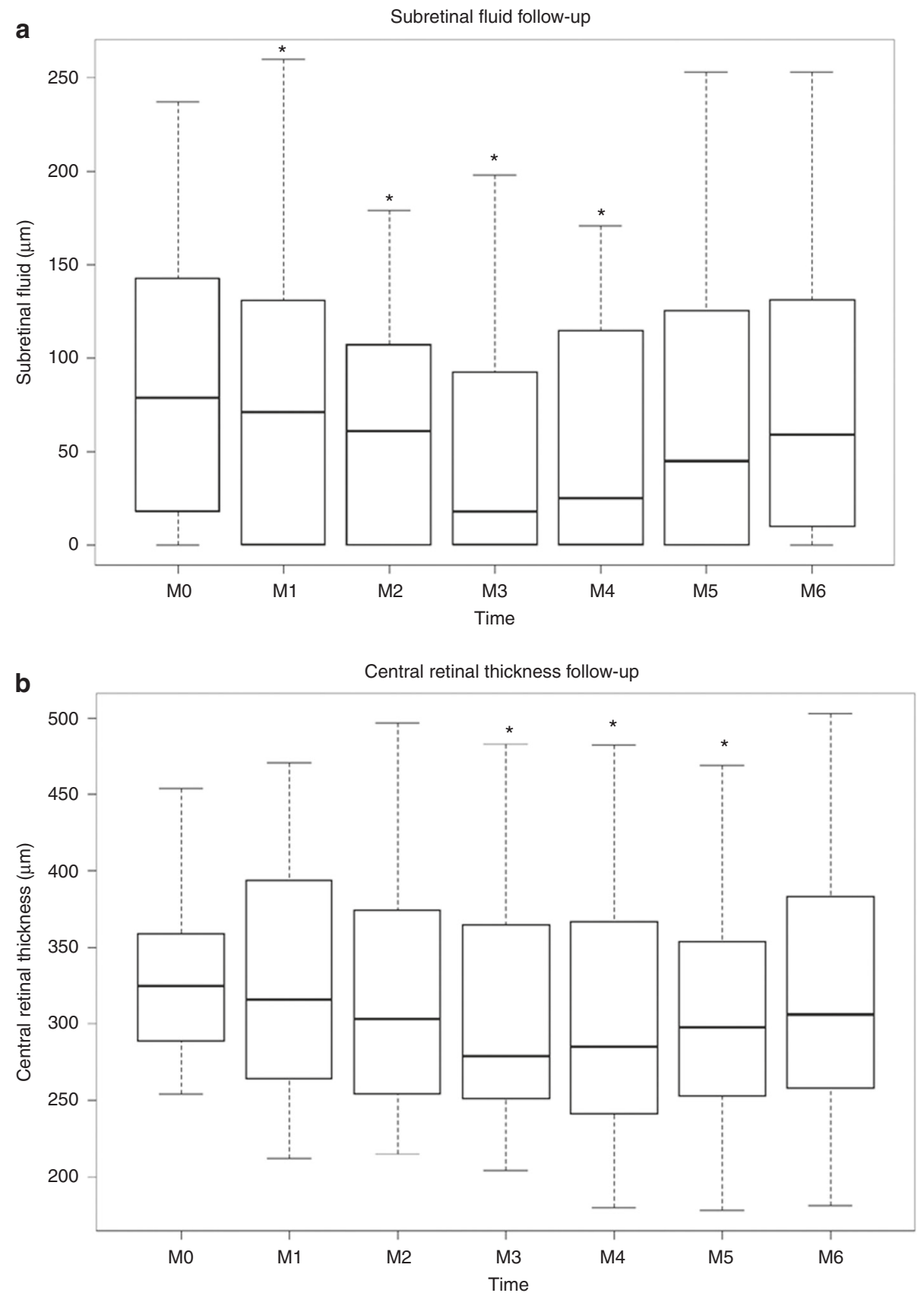

Fig. 1 Clinical results. a Relative changes in subretinal thickness (SRF, $\mu \mathrm{m})$ measured with Spectralis OCT during the study period, month $0(\mathrm{MO})$ to month 6 (M6). SRF thickness is significantly reduced as compared to M0, at M1 ( $p=0.012), M 2(p=0.040), M 3(p=0.014), M 4(p=0.024)$. b Relative change in central retinal thickness (CRT, $\mu \mathrm{m})$ automatic values with Spectralis OCT during the study period, month 0 (MO) to month 6 (M6). CRT is significantly reduced as compared to M0, at M3 $(p=0.011), M 4(p=0.007)$, and M5 $(p=0.028)$. At each time point (Month, M), the data have been summarized using box and whisker plots, the upper and lower $95 \% \mathrm{Cls}$ (confidence interval) are marked with the whiskers, the box represents the interquartile range, and the median is represented by the bold horizontal line bisecting the box. Paired Wilcoxon signed-rank tests were used. ${ }^{*} p<0.05$

anti-edematous effect unrelated to the CNV, as previously described in other retinal conditions ${ }^{23,26}$. To test the antiangiogenic potential of MRA on CNV, we used an experimental model induced by laser photocoagulation in rodents, a recognized model for $\mathrm{nAMD}^{33}$. The argon laser induces a rupture of the Bruch's membrane, which separates the RPE from the choroidal vasculature, leading to the growth of neovessels from the choroid toward the retina in about 2 weeks. The permeability and size of $\mathrm{CNV}$ were assessed in vivo by fluorescein angiography (FA) at day 14 and at day 16 after sacrifice by ex vivo staining with FITCisolectin on choroid flat-mounts. Spironolactone $(25 \mathrm{mg} / \mathrm{kg} / \mathrm{day}$, subcutaneous injections from day 0 to day 13) was as efficient as intravitreal anti-VEGF injection $(5 \mu \mathrm{l}$ at $1.5 \mu \mathrm{g} / \mu \mathrm{l}$, at day 0$)$ in reducing choroidal vascular leakage assessed by angiographic score and decreasing the volume of the CNV lesions measured on choroid flat-mounts (Fig. 2a). Co-administration of anti-VEGF and spironolactone showed an additive effect on neovascular permeability observed on FA as compared to anti-VEGF alone (Fig. 2a). Systemic spironolactone inhibited the accumulation of allograft inflammatory factor 1 (IBA1)-positive macrophages/ microglia in the laser burn area (Supplementary Fig. 5a) and reduced the expression of inflammatory cytokines (monocyte 
Table 1 Functional and structural outcome measures in nAMD patients treated with spironolactone

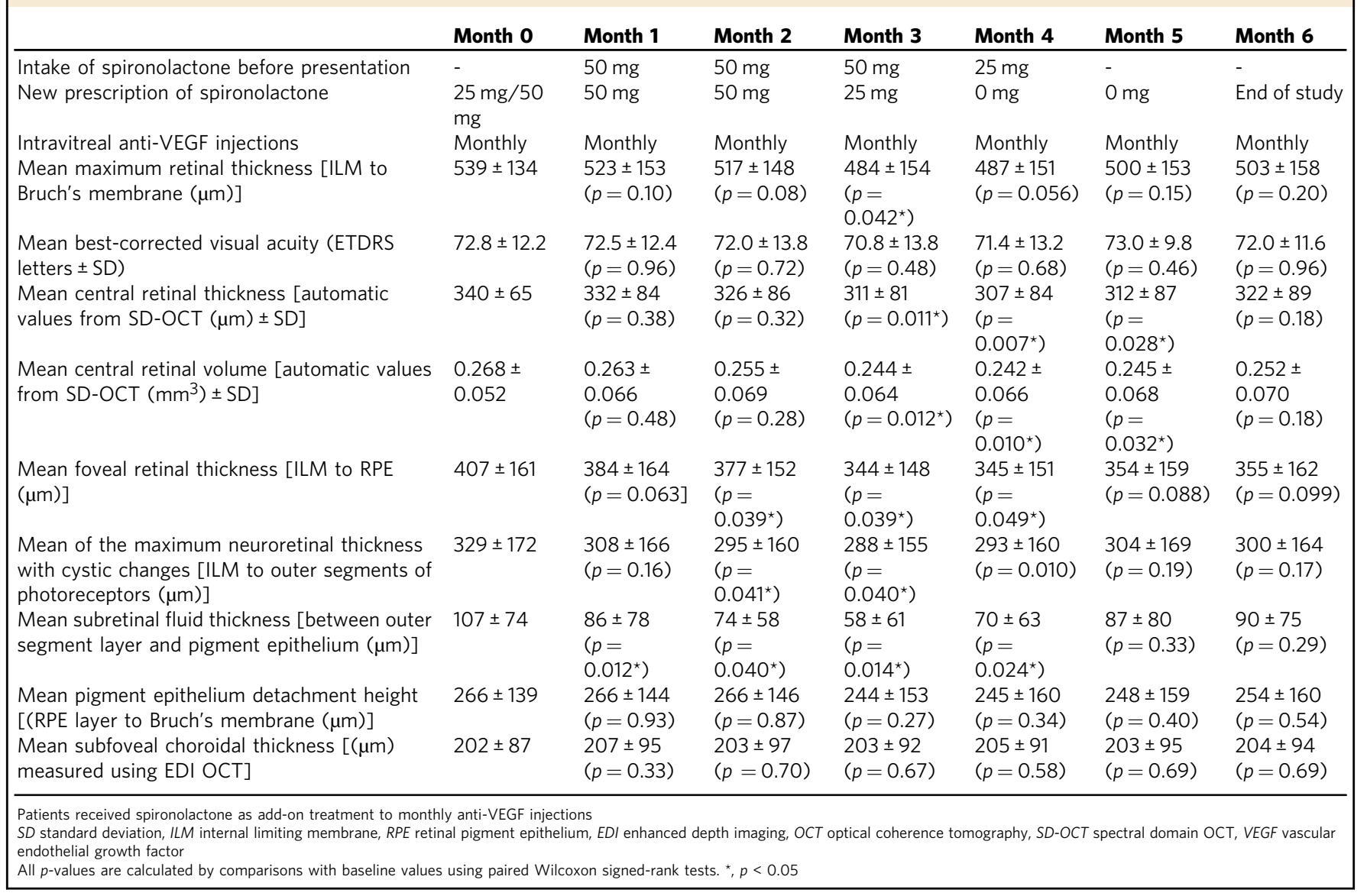

chemoattractant protein 1 [MCP-1], interleukin [IL] $1 \beta$, IL6, and tumor necrosis factor [TNF]) in the RPE-choroid complexes at day 3 but had no significant effect on the expression of angiogenic genes such as VEGF, PlGF, ANGPTL4, HIF1 $\alpha$, and TGF $\beta$ (Supplementary Fig. 5b). In the rat ocular media, the VEGF level was decreased significantly by anti-VEGF but not by spironolactone; conversely, the MCP-1 level was decreased by spironolactone but not by anti-VEGF (Supplementary Fig. $5 \mathrm{c}$ ). These results demonstrated the effect of pharmacological MR antagonism on macrophage/monocytes recruitment. Potential nonspecific hormonal effects of spironolactone on CNV was excluded as oral eplerenone (INSPRA ${ }^{\circledR}, 200 \mathrm{mg} / \mathrm{kg} /$ day, $0.2 \%$ in chow), a more specific MR antagonist without non-MR related hormonal side-effects ${ }^{34}$ had similar anti-angiogenic effects of rat CNV (Fig. 2b).

To test whether local spironolactone could be as efficient as systemic administration, we elaborated spironolactone-loaded poly(lactic-co-glycolic) acid (PLGA) microspheres(MSs), which can be injected into the vitreous and are well tolerated ${ }^{35}$. The mean size of the particles was $21.87 \pm 6.84$ (SD) $\mu \mathrm{m}$. Scanning electron microscopy revealed spherical particles with smooth surface (Supplementary Fig. 6a). The encapsulation efficiency of spironolactone was $77.03 \%(128.38 \mu \mathrm{g}$ spironolactone per $\mathrm{mg}$ MSs) and a constant release rate of $2.35 \mu \mathrm{g}$ spironolactone per $\mathrm{mg}$ MSs per day was achieved over 31 days (Supplementary Fig. 6a). A single injection $(5 \mu \mathrm{l}$ of $2.2 \mu \mathrm{g} / \mu \mathrm{l})$ of spironolactone-loaded MSs in the vitreous of rat eyes at day 0 inhibited choroidal neovascular permeability on FA and significantly reduced the volume of CNV at day 14, as compared to non-loaded-MS-injected eyes (Supplementary Fig. 6b). Therefore, systemic and local spironolactone showed similar anti-angiogenic effects on rat $\mathrm{CNV}$.
Endothelial MR inactivation reduces $\mathrm{CNV}$ in mice. To identify the cellular targets of MRAs, we evaluated the expression and localization of MR in the retinas of rat eyes developing CNV. MR expression was upregulated in the neural retina and in the RPEchoroid complex at day 3 after laser induction, and returned to the basal level at day 16, the late phase of CNV (Supplementary Fig. 7a). Immunohistochemistry showed that the MR was expressed not only in the ganglion cell layer, the inner nuclear layer, the RPE and endothelial cells as described previously ${ }^{22,23}$, but also in the infiltrating cells and in neovascular components within subretinal spaces surrounding the laser burns (Supplementary Fig. 7b-d, arrows). To determine which cell types could drive the MR-induced angiogenic signals, we used conditional transgenic models lacking a functional MR in specific cell types. Specific conditional ablation of MR in endothelial cells only (Vecadh-MR-KO) significantly reduced CNV on FA and on choroidal flat-mounts (Fig. 3b), demonstrating that endothelial cells drive MR-induced angiogenesis. This finding was further confirmed in another ocular model of angiogenesis, in which neovessel growth is induced in the avascular cornea by corneal de-epithelialization. In this model, angiogenesis was also significantly impaired in Vecadh-MR-KO mice (Fig. 3c), suggesting a broader angiogenic effect of the endothelial MR. Of note, targeted MR inactivation in smooth muscle cells (SMA-MR-KO mice) or in granulocytes and macrophages (Lys-MR-KO mice) has no effect on the development of CNV (Supplementary Fig. 8a, b), confirming the endothelial cell specificity of the MR function in angiogenesis. To validate this finding, we used another transgenic model in which $\mathrm{MR}$ is constitutively deleted under the endothelial Tie2 promoter (Tie2-MR-Ko), expressed both in endothelial cells and in myeloid cells ${ }^{36}$. Using the laser-induced 
a
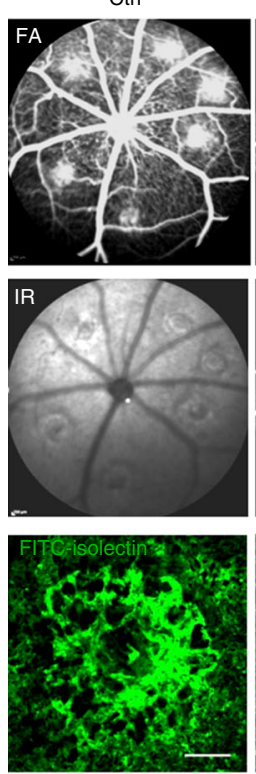

Incidence of $\mathrm{CNV}$ angiographic grades

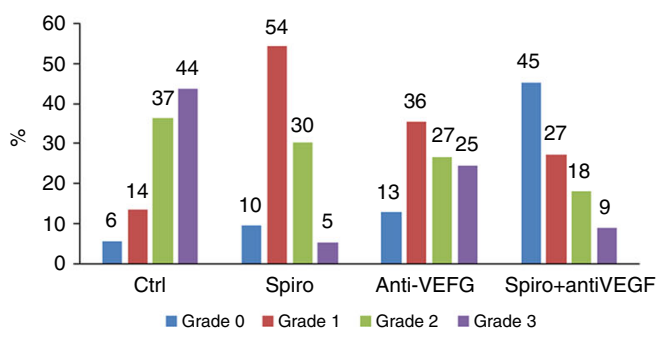

Anti-VEGF
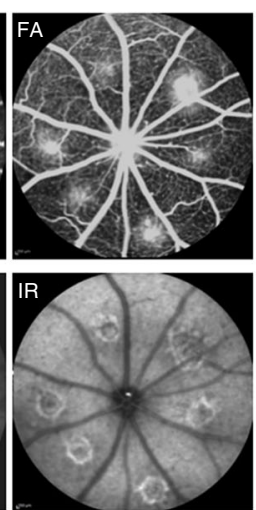

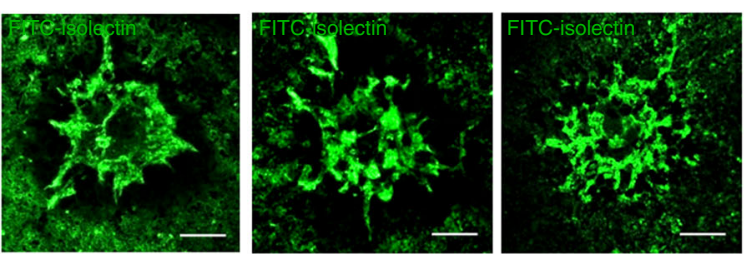

Spiro+anti-VEGF
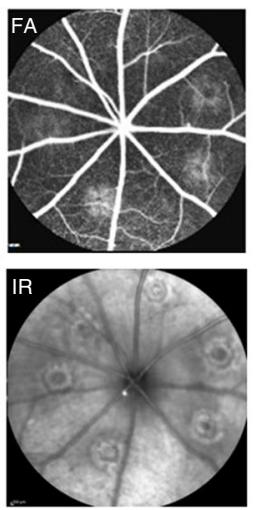
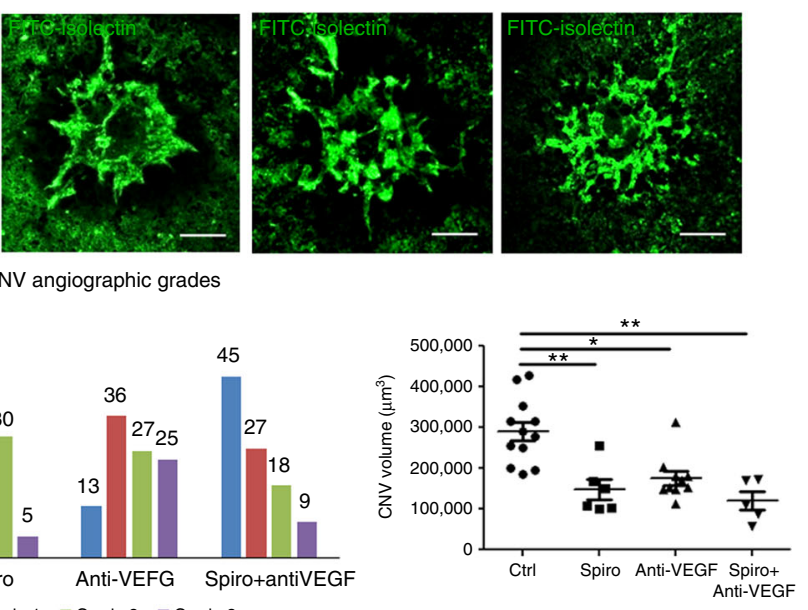

b
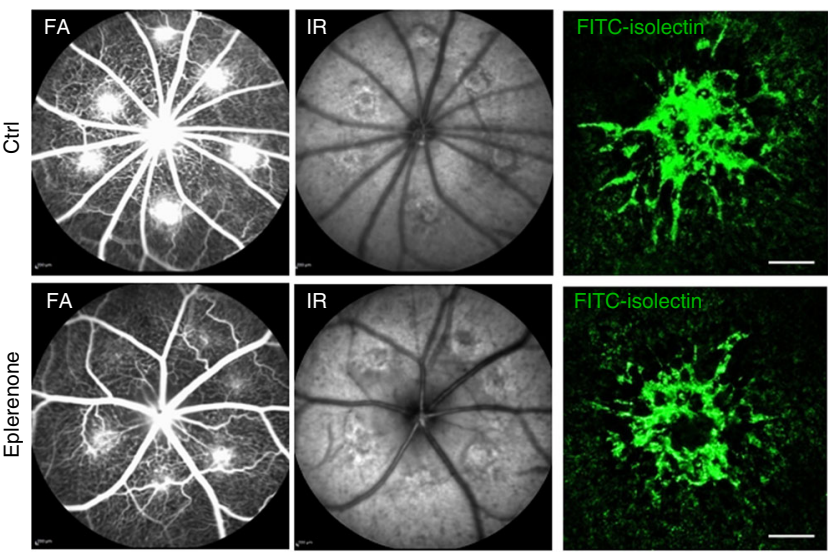

Incidence of CNV angiographic grades
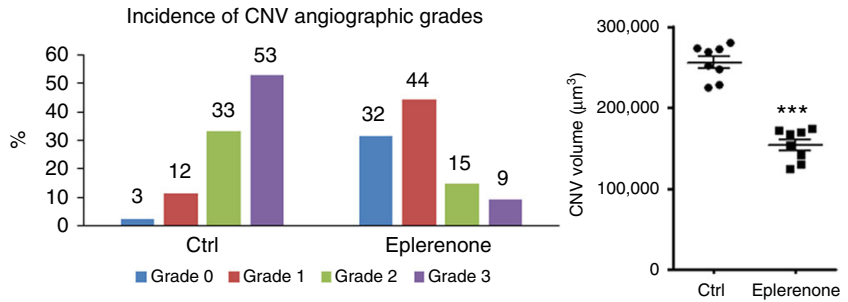

Fig. 2 Spironolactone and eplerenone reduce CNV in a rat nAMD model. a Spironolactone (Spiro) significantly reduces the CNV angiographic grades evaluated on fluorescein angiography (FA) $(p=0.0025)$ and the CNV volume labeled with FITC-isolectin (green) $(p=0.002)$ as compared to the control group (Ctrl). Infrared images (IR) are used to localize and check the efficient laser-induced burns. The effect of spironolactone is not different from antiVEGF in reducing the choroidal neovascular leakage on FA and inhibiting the CNV in rat choroidal flat-mounts. Combining the two treatments allows an enhanced effect in reducing vascular permeability compared to anti-VEGF alone $(p=0.0335)$. $\mathbf{b}$ Eplerenone, a more specific MR antagonist, given orally, significantly reduces CNV angiographic grades $(p=0.0012)$ and CNV volumes $(p<0.0001)$. Bars: $100 \mu \mathrm{m}$. FA Data are expressed as the incidence of CNV angiographic grades of the total laser impacts in each group. CNV volumes are expressed as mean \pm SEM of average CNV size per rat. $n$ represents the number of rats. Linear mixed model was used for statistical analyses. ${ }^{\star} p<0.05,{ }^{\star \star} p<0.01,{ }^{\star \star \star} p<0.001$ 

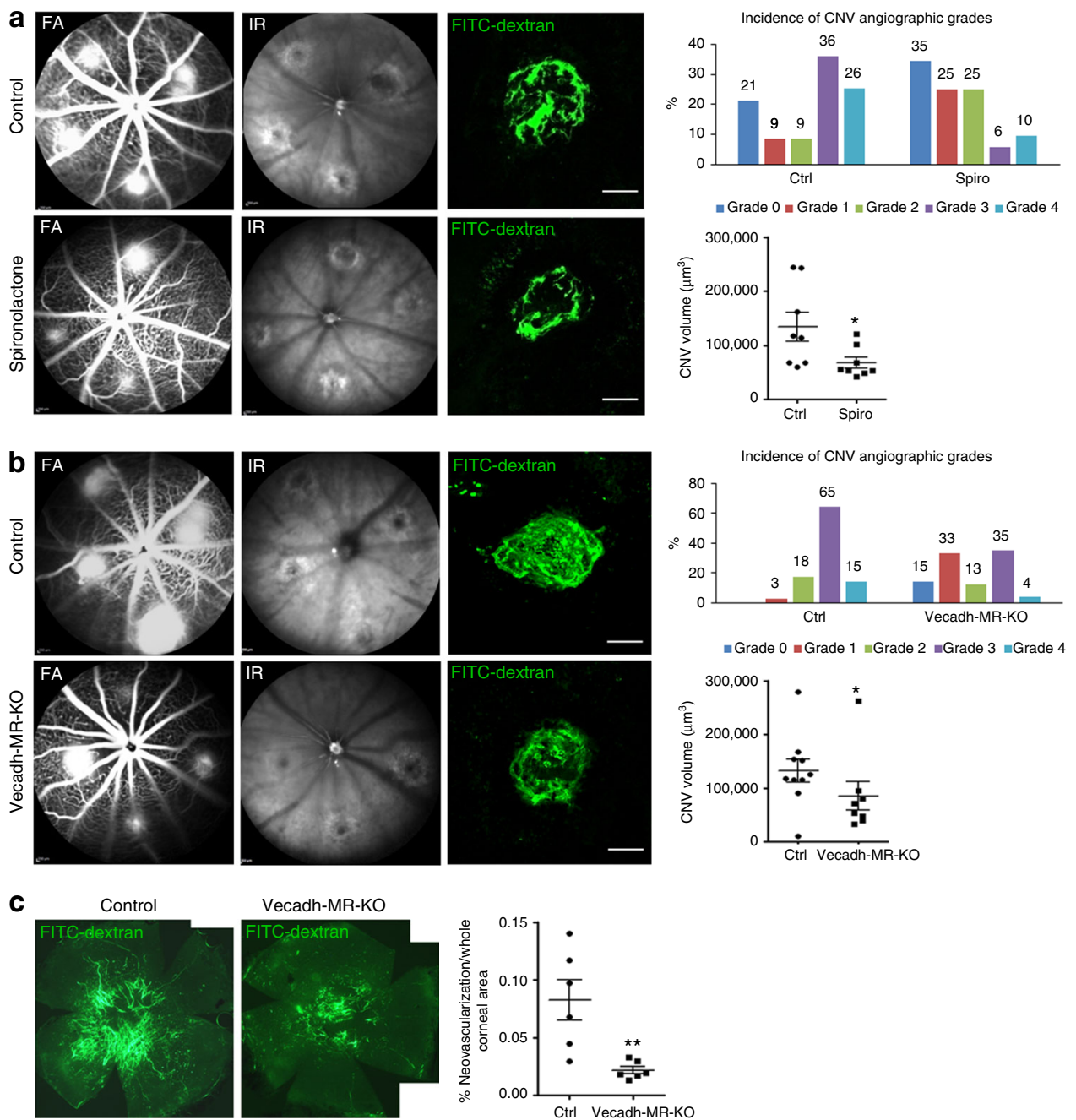

Vecadh-MR-KO
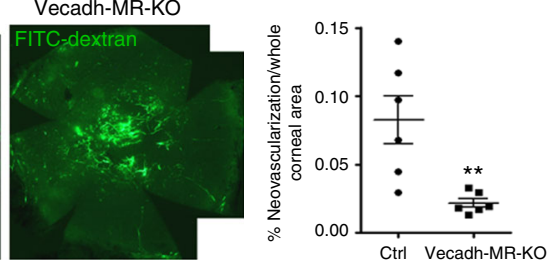

Fig. 3 Vascular endothelial MR contributes to CNV. a Systemic spironolactone significantly reduces CNV fluorescein angiographic (FA) grades ( $p<0.001)$ as well as CNV volume $(p=0.0348)$ as compared to control mice. CNV were labeled with FITC-dextran (green). Infrared (IR) images show up all the laser burns in the fundus. $\mathbf{b}$ Cell-type-specific MR deletion from endothelial cells using the VE-Cadherin promoter (Vecadh-MR-KO) reduces CNV leakage on FA $(p=0.0041)$ and decreases the volume of CNV labeled with FITC-dextran (green) in mice $(p=0.0434)$. IR images show up all the laser burns. Bar: $100 \mu \mathrm{m}(\mathbf{a}, \mathbf{b})$. FA Data are expressed as the incidence of CNV angiographic grades of the total laser impacts in each group. CNV volumes are expressed as mean \pm SEM of the average CNV size per mouse. $n$ represents the number of mice. Linear mixed model was used for statistical analyses. ${ }^{*} p<0.05$. $\mathbf{c}$ In a model of corneal neovascularization using Vecadh-MR-KO mice, a reduction in corneal neovessels labeled with FITC-dextran was observed compared to control mice. Quantification of the neovascular surface on mosaic images confirms a significant decrease in the neovascularization/whole corneal area ratio in Vecadh-MR-KO mice. Data are expressed as mean \pm SEM. $n$ represents the number of mice. Non-parametric Mann-Whitney $U$-test was used. ${ }^{\star \star} p<0.01$

nAMD model, the angiographic score and the CNV volume were also reduced in Tie2-MR-KO mice (Supplementary Fig. 8c), however, the effect was not significantly different from that observed in Vecadh-MR-KO mice ( $p=0.1740$, repeated measures linear mixed model), suggesting that myeloid MR is not essential in laser-induced CNV. In addition, systemic treatment with spironolactone (Fig. 3a), which antagonizes MR in all cells did not result in a more potent anti-angiogenic effect in mice as compared to the effect observed in Vecadh-MR-KO mice $(p=0.5507$, repeated measures linear mixed model). Lack of effect on CNV in SMA-MR-KO, which is tamoxifen-inducible, eliminates a potential non-specific effect of Cre with tamoxifen. A non-specific effect of tamoxifen alone was eliminated since control mice received tamoxifen when used to activate the Cre recombinase in the Vecadh-MR-KO mice, and because in the Tie2-MR-KO mice, which is not tamoxifen-inducible, the reduction in CNV was not higher than in the Vecadh-MR-KO mice. Taken together, these results suggest that endothelial MR is required for the development of CNV.

Decorin mediates angiogenic effect of endothelial MR in CNV. Among the potential molecular targets of the MR in the endothelial cells that could regulate angiogenesis, decorin (DCN) was selected from a full transcriptomic approach designed to identify genes regulated by aldosterone in the whole rat retina consisting of neural retina and RPE-choroid complex (Supplementary data 1). The expression of DCN messenger RNA (mRNA), encoding a matrix protein of the member of the small leucinerich proteoglycan family, was down-regulated by approximately 2.64 times in the transcriptome of the rat whole retina injected with aldosterone ( $20 \mathrm{nM}$ final vitreous concentration) compared with that of the vehicle-injected one (Supplementary Data 2). In the rat $\mathrm{CNV}$ model, DCN protein expression was reduced in the 
a
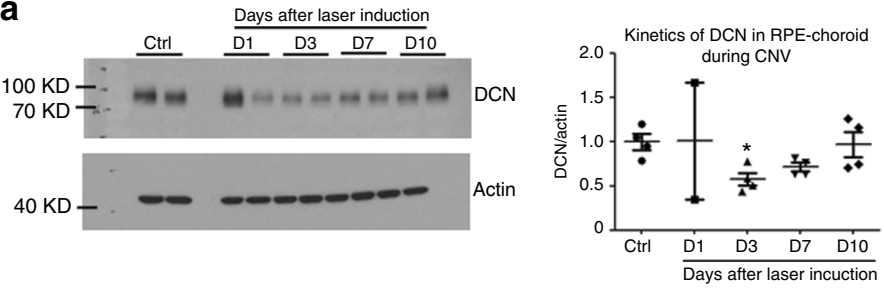

b
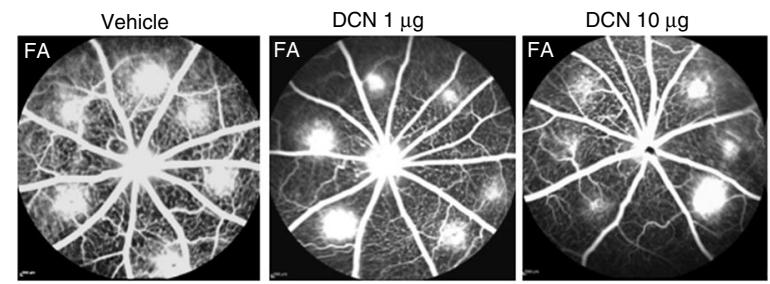

Incidence of CNV angiographic grades
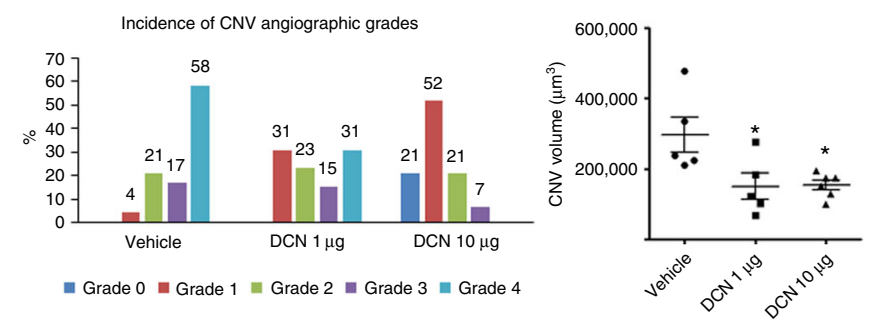

C

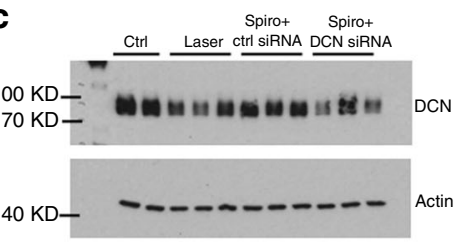

d
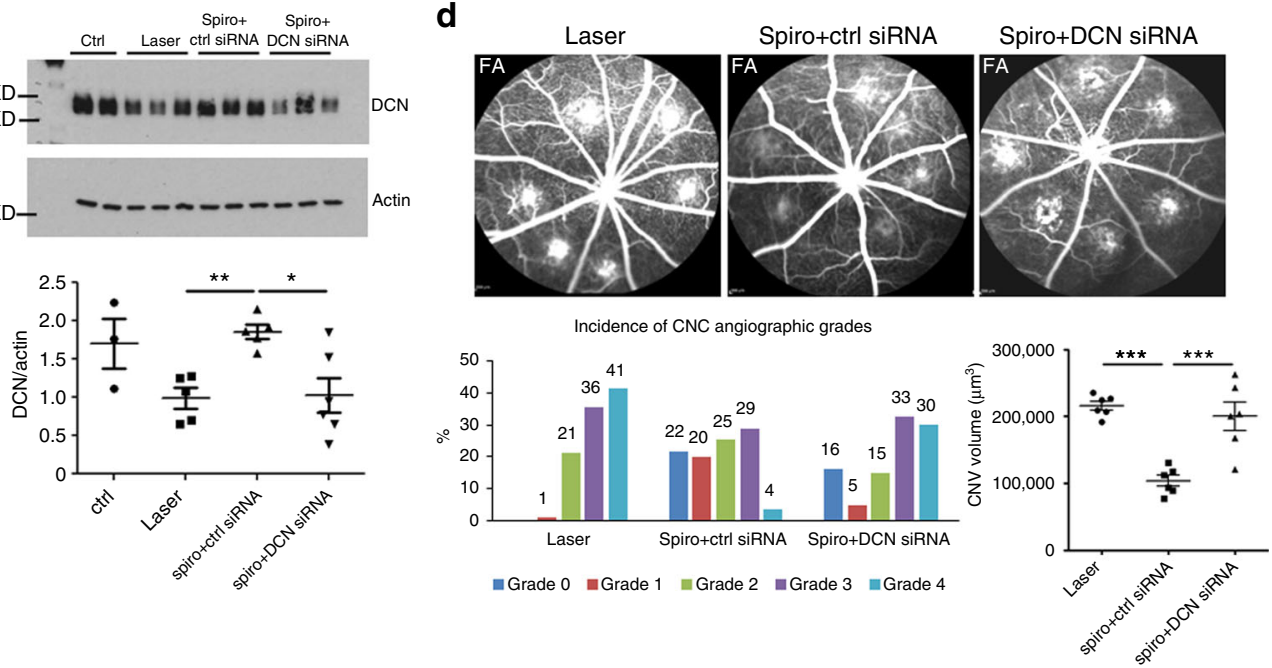

Fig. 4 Spironolactone inhibits CNV through induction of anti-angiogenic decorin protein. a On western blot, the decorin (DCN) level decreases in the rat retinal pigment epithelium (RPE)-choroid at different time point (day 1, 3, 7, and 10) after laser induction compared to the normal rat RPE-choroid (ctrl). Densitometric quantification shows significant decrease of DCN protein in rat RPE-choroid at day 3 after laser induction. $\mathbf{b}$ Intravitreal injection (IVT) of recombinant $\mathrm{mDCN}$ protein in rat eyes inhibits choroidal vascular leakage on fluorescein angiography (FA); DCN $10 \mu \mathrm{g} / \mathrm{ml}$ significantly reduces the CNV angiographic grades $(p=0.02)$, whereas DCN at both $1 \mu \mathrm{g} / \mathrm{ml}(p=0.0461)$ and $10 \mu \mathrm{g} / \mathrm{ml}(p=0.0388)$ decrease the size of CNV induced by laser. c IVT of DCN siRNA in rat eyes with laser-induced CNV. Treatment with spironolactone (Spiro) in the presence of control siRNA significantly increases the DCN protein level in the rat RPE-choroid $48 \mathrm{~h}$ after laser induction. IVT of DCN siRNA prevents the increase in the DCN protein induced by spironolactone. d Treatment with spironolactone in the presence of control siRNA inhibits choroidal neovascular leakage on FA at day 14 after laser induction ( $p=0.0194)$. IVT of DCN siRNA at day 0 and 3 after laser induction abrogates the effect of spironolactone on vascular leakage $(p=0.0344)$. Spironolactone in the presence of control siRNA reduces significantly CNV volume as compared to laser control $(p<0.0001)$. DCN siRNA injected at day 0 and 3 after laser induction abrogates the effect of spironolactone on CNV volume $(p=0.0003)$. Western blot data are expressed as mean \pm SEM. Dots represent individual RPE-choroid sample. Non-parametric Kruskal-Wallis test was used. FA Data are expressed as the incidence of CNV angiographic grades of the total laser impacts in each group. CNV volumes are expressed as mean \pm SEM of the average CNV size per rat. $n$ represents the number of rats. Linear mixed model was used for statistical analyses. ${ }^{\star} p<0.05,{ }^{\star \star} p<0.01,{ }^{\star \star \star} p<0.001$

RPE-choroid as early as 3 days after CNV induction (Fig. 4a) consistent with the timing of MR upregulation in the RPEchoroid (Supplementary Fig. 7a). The DCN levels partly recovered from day 7 to day 10 (Fig. 4a), suggesting that decreased DCN might contribute to the pro-angiogenic balance in this CNV model. To analyze the anti-angiogenic effect of DCN in CNV, we used a recombinant mouse DCN protein that shares $87 \%$ aminoacid sequence identity with rat DCN. Intravitreous injection of recombinant $\mathrm{mDCN}$ was performed after laser induction at an estimated final concentration of $1 \mu \mathrm{g} / \mathrm{ml}$ or $10 \mu \mathrm{g} / \mathrm{ml}$ in rat 
vitreous. At day 14, eyes treated with recombinant $\mathrm{mDCN}$ at $10 \mu \mathrm{g} / \mathrm{ml}$ showed reduced choroidal vascular leakage (Fig. 4b). The CNV volume was decreased significantly by DCN at both 1 $\mu \mathrm{g} / \mathrm{ml}$ and $10 \mu \mathrm{g} / \mathrm{ml}$ (Fig. $4 \mathrm{~b}$ ), confirming a major role of DCN in inhibiting $\mathrm{CNV}$ proliferation and leakage. In the rat nAMD model, DCN expression and protein were reduced at day 3 after laser induction and spironolactone upregulated the DCN transcripts (Supplementary Fig. 9a) and the DCN protein levels (Supplementary Fig. 9b) in the rat RPE-choroid, suggesting that the anti-angiogenic effect of spironolactone could be mediated by DCN expression. To test this hypothesis, we designed a strategy to interfere with the effect of spironolactone on DCN expression. We co-administered systemic spironolactone with intraocular siRNA directed against DCN. Spironolactone (in the presence of scrambled siRNAs) significantly prevented the laser-induced decrease in DCN protein at day 2 following induction (Fig. 4c). In contrast, DCN siRNA inhibited the DCN upregulation by spironolactone (Fig. 4c), confirming its efficiency in DCN knockdown. We next tested whether abrogation of the DCN upregulation induced by spironolactone could clinically reduce the anti-angiogenic effect of spironolactone. Rats were treated daily with spironolactone and injected intravitreously with scrambled or DCN siRNA at day 0 and day 3. At day 14 , coadministration of spironolactone and DCN siRNA abolished the effect of spironolactone on choroidal neovascular permeability as estimated by FA (Fig. 4d) and reduced the CNV volume (Fig. 4d), demonstrating that DCN mediates, at least in part, the antiangiogenic effect of spironolactone.

Taken together, these results show that the clinical effect of spironolactone on nAMD refractory to anti-VEGF can be explained by a direct antagonism of the MR in endothelial cells, leading to DCN overexpression.

\section{Discussion}

Anti-VEGF therapy has revolutionized the visual prognosis of $\mathrm{CNV}$, in particular in nAMD patients, from rapid progression to central blindness to central vision stabilized or even improved for several years ${ }^{12}$, demonstrating the key role of VEGF in the regulation of hydro-ionic retinal homeostasis and vascular permeability ${ }^{37}$. However not all nAMD patients respond well to anti-VEGF treatment, particularly when $\mathrm{CNV}$ develops underneath the detached retinal pigment epithelium, which represents more than $50 \%$ of CNV in AMD cases (Type $1 \mathrm{CNV})^{38,39}$ and complicates $30-40 \%$ of chronic CSCR cases. Moreover, in around $50 \%$ of nAMD patients, depending on the drug and regimens, fluid retention persists in the macula despite optimal anti-VEGF treatments ${ }^{18,40}$. Anti-platelet-derived-growth-factor (PDGF) therapies failed to demonstrate synergistic effects with anti-VEGFs in 2 phase- 3 clinical trials ${ }^{41}$, and no other therapeutic options are currently available for nAMD patients not responding optimally to anti-VEGFs. In our clinical pilot study, despite long-lasting anti-VEGF-resistant macular edema, patients treated orally with the MRA spironolactone for 4 months while continuing monthly injection of the same antiVEGF treatment, showed a significant reduction in signs of CNV activity as measured by FA and SD-OCT. Notably, the effect was lost when spironolactone was stopped, demonstrating that the clinical improvement was an effect of the spironolactone. Despite anatomical improvement, no immediate effect on visual acuity was observed in this group of chronic nAMD patients, which could be due to disease chronicity and irreversible lesions and/ or the short duration of treatment, or sub optimal route of administration. In a recent retrospective study without a control group or controlled time after MRA application, a beneficial effect of MRA on subretinal fluid absorption in anti-VEGF refractory nAMD was also reported ${ }^{42}$. Whether the MRA acted on fluid reabsorption only or also on neovessels could not be determined.

No animal model recapitulates all nAMD features, and the common laboratory animals have no macula. However, laserinduced $\mathrm{CNV}$ models in rodents and primate are validated in screening anti-angiogenic drugs for $\mathrm{nAMD}^{33}$. In the present study, the MRA spironolactone administrated systemically in rats was as efficient as the reference intravitreal anti-VEGF drug for suppressing CNV. Eplerenone, a more specific MR antagonist, given orally, also efficiently reduced rat $\mathrm{CNV}$, supporting the specific role of MR pathway activation in choroidal neovascularization. Spironolactone significantly inhibited the development of CNV in rats without affecting the ocular VEGF levels; however, MCP-1 expression and microglia/macrophage infiltration at the site of $\mathrm{CNV}$, both known contributors to $\mathrm{CNV}$ in $\mathrm{AMD}^{43,44}$, were reduced. The monocyte-expressed MR has been reported to stimulate pro-inflammatory macrophages ${ }^{45,46}$. However, MR deletion specifically in myeloid cells did not prevent CNV, and MR deletion in both endothelial cells and myeloid cells did not show a superior inhibitor effect on $\mathrm{CNV}$ as compared to MR deletion in endothelial cells only, demonstrating that the benefit of MRA in $\mathrm{CNV}$ is not primarily mediated by MR modulation of the macrophage phenotype. In contrast, specific deletion of the MR in endothelial cells significantly inhibited choroidal and corneal neovascularization, indicating that $\mathrm{MR}$ activation is proangiogenic in wound healing. In addition, systemic spironolactone treatment in mice, which antagonizes all MR expressing cells, did not exert superior anti-angiogenic effect than the deletion of MR specifically in endothelial cells (Vecadh-MR$\mathrm{KO})$. Similar anti-angiogenic effects of spironolactone have been reported in a model of oxygen-induced retinopathy in which neovessels develops from the retinal vasculature ${ }^{32}$.

The search for molecular pathways regulated by MR potentially modulating angiogenesis and inflammation led us to identify $\mathrm{DCN}^{47}$ as a potential mediator of the MR effect. In fact, DCN was downregulated in the retina and RPE-choroid complex of aldosterone-injected rats. Furthermore, we showed an antiangiogenic effect of DCN on CNV. Importantly, laser-induced CNV decreased DCN expression in the RPE-choroid, and this effect was prevented by spironolactone treatment. In addition, a DCN siRNA suppressed the anti-angiogenic effect of spironolactone, identifying DCN as a novel molecular target of spironolactone. In the healthy human eye, DCN is strongly expressed in the cornea, where it intervenes in fibrillogenesis and tissue repair ${ }^{48}$. In the retina, $\mathrm{DCN}$ has been identified to be located in all layers but is particularly abundant in Bruch's membrane, which separates the RPE from the choroid, and in the choroid itself ${ }^{49}$. As a component of the extracellular matrix, DCN can modulate angiogenesis through interaction with numerous molecules ${ }^{47,50}$. In corneal wound healing models and in inflammatory angiogenesis, DCN is anti-angiogenic ${ }^{51,52}$. This effect results from the sequestration of growth factors such as TGF- $\beta$, an antagonism of tyrosine kinase family receptors including the VEGF-R2 ${ }^{50,53}$, and stimulation of angiostatic molecules such as thrombospondin and tissue inhibitor of metalloproteinase 3 (TIMP3) ${ }^{54}$. Under hypoxic conditions, DCN reduced choroidal endothelial cell proliferation induced by the RPE in culture ${ }^{55}$. In addition, DCN exerts an anti-inflammatory effect through a decrease in macrophage proliferation ${ }^{56}$ and recruitment, in turn mediated by MCP-1 downregulation ${ }^{57}$. In mice CNV, DCN inhibited TGF $\beta$-induced $\operatorname{smad} 2 / 3$ pathway ${ }^{58}$. DCN expression in relation to AMD has not been studied specifically; however, a quantitative proteomic analysis of Bruch's membrane/choroid from AMD eyes showed significantly decreased DCN levels compared to those in healthy controls ${ }^{59}$. In addition, DCN 
expression was reduced by approximately $20 \%$ in advanced wet as compared to advanced dry AMD eyes ${ }^{59}$, supporting a role for DCN in wet AMD.

In the retina, experimental MR activation has been shown to be pathogenic leading to choroidal vessel dilation, leakage and subretinal fluid retention (mimicking CSCR) ${ }^{23}$, retinal edema mediated by glial hydro-ionic deregulation ${ }^{22}$ and retinal neovascularization in a model of retinopathy of prematurity ${ }^{32}$. In the rodent retina, MR and hydroxysteroid dehydrogenase type 2 are co-expressed in several cell types but not all suggesting that MR pathway could thus be activated by both gluco and mineralocorticoids ${ }^{23}$. Based on clinical observations, MRAs are currently used to reduce edema in $\operatorname{CSCR}^{24,60}$. In the present study, we demonstrated that MR activation in vascular endothelial cells contributes to $\mathrm{CNV}$, which is also frequently associated with CSCR. The MR pathway may thus be a common mechanism involved in CNV development in AMD and in CSCR. Why MR is hyper-activated in these conditions is not fully understood. In most organs, the MR are occupied by glucocorticoids, prevalent in the plasma over aldosterone ${ }^{61}$. In nAMD, MR activation may explain the poor efficacy of glucocorticoids on $\mathrm{CNV}^{11}$, with their potent anti-inflammatory effects being overridden by the proedematous and pro-angiogenic MR pathway. Further studies are required to analyze the MR/GR pathway in AMD.

Local delivery is the method of choice for ocular diseases, allowing higher ocular drug levels without systemic side-effects. For this purpose, slow-release drug delivery systems are mandatory to reduce the frequency of ocular injections. The pre-clinical tolerance of our polymeric spironolactone MS formulation has been demonstrated previously ${ }^{35}$ offering a basis for further clinical testing.

In conclusion, our clinical and experimental data demonstrate that vascular MR activation contributes to CNV. In a pilot study, including patients with refractory and long-lasting exudative signs despite anti-VEGF treatment, we showed that MRA might have an effect on intra and/ or subretinal fluid. In addition, our experimental data support that MR pathway activation contributes to the pathogenesis of nAMD through a VEGFindependent pathway related to DCN, which represents a therapeutic target for nAMD. Further randomized clinical trials, optimally using ocular dedicated formulations, are required to evaluate the therapeutic potential of MR pathway blockade in patients with refractory nAMD.

\section{Methods}

Patients. A prospective clinical pilot study was performed to evaluate in vivo the effect of spironolactone, an oral MRA, in patients with exudative signs of nAMD. This study was approved by the local ethics committee (\#184/14 ethical committee approval, Canton de Vaud), and performed following the regulations of the mentioned committees. It adhered to the Declaration of Helsinki for human research. Informed consent was obtained from all human participants. This study has been retrospectively registered on 16 November, 2018 under the registration number NCT03744767.

Only patients with refractory stable intraretinal or subretinal fluid present for $\geq 6$ months in a row on monthly SD-OCT despite monthly intravitreal of the same anti-VEGF treatment (ranibizumab or aflibercept) were included. The total duration of anti-VEGF treatment before inclusion in the study was required to be $\geq 12$ months to avoid the effect of improvement due to treatment initiation. The thickest macular A-scan from the inner limiting membrane to the RPE band was required to be $\geq 350 \mu \mathrm{m}$. Patients giving informed consent were included from September 2014 to March 2015.

The exclusion criteria were polypoidal choroidal vasculopathy, poor imaging quality of the fundus, or any contra-indication to systemic spironolactone (arterial pressure $>160 / 100, \mathrm{~K}+>5.0 \mathrm{mmol} / \mathrm{l}, \mathrm{Na}+<135 \mathrm{mmol} / \mathrm{l}$, calculated creatinine clearance under $30 \mathrm{ml} / \mathrm{min}$ [coefficient $\times\{140$-age $\} \times$ weight $/$ serum creatinine; coefficient $=1.23$ for males and 1.04 for females], acute renal failure, renal dialysis, unspecified renal problem, arrhythmia, cardiovascular polymorbidity with thromboembolic risk, known hypersensitivity to spironolactone, ongoing treatment with epleronone, and decompensated hepatic cirrhosis). The treating physician was informed and potentially interacting medications were discussed. During the 6 months study, participants continued fixed monthly intravitreal injections of the same anti-VEGF drug as before, and were prescribed adjunctive oral spironolactone for 4 months $(25 \mathrm{mg}$ /day in week 1 , then $50 \mathrm{mg}$ /day until Month 3 , decreasing to $25 \mathrm{mg}$ /day during Month 4, then stopped) and followed off spironolactone until Month 6. Safety monitoring included history taking for adverse events, arterial blood pressure and laboratory analysis for changes in serum levels of $\mathrm{K}+, \mathrm{Na}+$, creatinine, and urea. In case of clinically significant changes, the investigator was allowed to reduce the dosage of spironolactone. At each monthly follow-up visit during the 6 months study, the best-corrected visual acuity (as determined by the Early Treatment Diabetic Retinopathy Study [ETDRS] test score) was recorded and SD-OCT was performed using the follow-up mode. The SD-OCT cubes included 49 horizontal B-scans, encompassing a $6 \times 6 \mathrm{~mm}$ retinal area, centered on the CNV lesion. The predetermined primary endpoint was the retinal thickness change from baseline to Month 4 (end of adjunctive

spironolactone treatment) at the site of maximum retinal thickness (from internal limiting membrane to Bruch's membrane) measured at baseline and each followup visit on the identical A-scan using the inbuilt Spectralis follow-up software. Secondary endpoints included the change in maximum retinal thickness from month 4 to month 6 (anti-VEGF only), and changes in visual acuity (ETDRS letters), automated central retinal thickness (CRT) measurements, automated central retinal volume, the foveal retinal thickness, subretinal fluid, neuroretinal thickness, pigment epithelium detachment height, and choroidal thickness at each monthly evaluation time points.

All quantitative follow-up parameters were statistically analyzed and compared with the baseline parameters using paired Wilcoxon signed-rank tests. For data analysis, Microsoft Excel 2010 and JMP software for Windows (version 8.0.1, SAS Institute Inc., Cary, NC) were used. A two-tailed probability of 0.05 or less was considered statistically significant. The required study sample size was estimated before study initiation. According to the available literature on CSCR, and assuming that the treatment would be less effective in eyes with nAMD, our goal was to examine whether spironolactone could produce a minimum decrease of $15 \%$ in all measured retinal thicknesses. Therefore, to achieve a $90 \%$ power for detecting this degree of change, we estimated that 21 eyes would be required.

Microsphere elaboration. PLGA ratio 50:50 (Resomer ${ }^{\circledR} 503$ ) was purchased from Boehringer Ingelheim Pharma GmbH \& Co. (Ingelheim, Germany). Spironolactone was obtained from Sigma-Aldrich (Schnelldorf, Germany). Polyvinyl alcohol 72,000 g/mol (PVA) was obtained from Merck KGaA (Darmstadt, Germany). All organic solvents were high performance liquid chromatography (HPLC) grade and used as received.

Spironolactone-loaded and non-loaded PLGA MSs were elaborated using an oil-in-water $(\mathrm{O} / \mathrm{W})$ emulsion solvent evaporation technique ${ }^{35}$. Briefly, The Ophase was prepared by suspension of $40 \mathrm{mg}$ of spironolactone in $1 \mathrm{ml}$ of PLGA solution in methylene chloride $(20 \% \mathrm{w} / \mathrm{v})$ resulting in a Spiro:PLGA ratio of 2:10 This phase was emulsified with the $\mathrm{W}$-phase composed by $5 \mathrm{ml}$ of PVA MilliQ ${ }^{\oplus}$ water solution $(2 \% \mathrm{w} / \mathrm{v})$. The emulsification was performed at 5,000 rpm for $1 \mathrm{~min}$ (Polytron ${ }^{\circledast}$ RECO, Kinematica GmbH PT 10-35, Lucerna, Switzerland). This O/W emulsion was subsequently poured onto $100 \mathrm{ml}$ of an aqueous PVA solution $(0.1 \%)$ and maintained under constant stirring for $3 \mathrm{~h}$ to allow MSs hardening. After that the MSs were washed, filtered, freeze-dried, and kept at $-20^{\circ} \mathrm{C}$ under dry conditions until used.

Microsphere characterization. Production yield percentage (PY\%) was calculated as the percentage of MS weight divided by the total amount of PLGA and spironolactone initially used in the formulation process.

$\mathrm{PY} \%=($ weight of microspheres/initial amount of PLGA and spironolactone $) \times$ 100

The mean particle size and particle size distribution were measured in aqueous suspension by light scattering in a Microtrac ${ }^{\circledast}$ S3500 Series Particle Size Analyzer (Montgomeryville, PA, USA).

The external morphology of MSs was evaluated by scanning electron microscopy (Jeol, JSM-6335F, Tokyo, Japan). Particles were gold sputter-coating for observation.

Spironolactone quantification. For in vitro characterization, spironolactone was quantified by HPLC ${ }^{35}$. Encapsulation efficiency of spironolactone in the microspheres was determined as follows: $5 \mathrm{mg}$ of MSs were dissolved in methylene chloride $(1 \mathrm{ml})$. After that, the polymer was precipitated by addition of $4 \mathrm{ml}$ of ethanol. The polymer suspension was vortex mixing and centrifuged $(8500 \mathrm{x} g$; $5 \mathrm{~min})$. The supernatant was recovered and filtered $(0.22 \mu \mathrm{m})$ for drug quantification by HPLC.

In vitro release studies were performed using 18 samples, which were prepared by suspension of $5 \mathrm{mg}$ spironolactone-loaded MSs in $1.8 \mathrm{ml}$ of release medium (phosphate buffered saline $\mathrm{pH} 7.4$ isotonic with $\mathrm{NaCl}$ ). Samples were kept at $37^{\circ} \mathrm{C}$ under constant agitation at $100 \mathrm{rpm}$ (Clifton Shaking Bath NE5, Nikel Electro Ldt, Avon, UK). At pre-set time point $(4,7,11,14,18,21,25,28$, and 31 days) all the samples were centrifuged $\left(8500 \mathrm{xg} ; 3 \mathrm{~min} ; 20^{\circ} \mathrm{C}\right)$. At each time point, two of the pellets obtained after centrifugation were frozen and freeze-dried. The amount of the drug remaining in the particles was quantified from the obtained powders using the same protocol described for encapsulation efficiency and the amount of released drug was calculated as the difference between the initial content and the 
actual content at each time point. The rest of the supernatants were removed and replaced with the same volume of fresh medium, to continue the release test.

Animals. All experiments were performed in accordance with the European Communities Council Directive 86/609/EEC and French national regulations and approved by local ethical committees (\#2541-2015110210279792 v3, Charles Darwin). Six to eight-week old male Long Evans rats from the Janvier Breeding Center (Le Genest-Saint-Isle, France) were used to create a rat model of CNV. Three-month old male mice with cell-type-specific MR deletion in endothelial, smooth muscle and myeloid cells, (i.e., Vecadh-MR-KO, Tie2-MR-KO, SMA-MR$\mathrm{KO}$ and Lys-MR-KO mouse models, respectively), were generated in the C57BL/6 genetic background as previously described ${ }^{62-64}$. Floxed MR (MRf/f) mice ${ }^{65}$ (kindly provided by Dr. Berger, Heidelberg, Germany) were crossed with mice expressing an inducible Cre-ERT2 recombinase driven by the VE-Cadherin promoter (Cdh5(PAC)-Cre-ERT2 line, kindly provided by Prof. Adams, London, UK; ${ }^{66}$ to generate Vecadh-MR-KO mice) or by the -SMA promoter ${ }^{67}$ (kindly provided by Dr. Metzger, Strasbourg, France; to generating SMA-MR-KO mice) Cell-specific MR inactivation was induced by tamoxifen injection $(1 \mathrm{mg} /$ day in corn oil for 5 consecutive days) 14 days before experiments and CNV induction. Both $\mathrm{KO}$ and control mice received same tamoxifen regimen.

Lys-MR-KO mice were obtained by mating mice expressing Cre recombinase in myeloid cells (LysMcre; ${ }^{63}$ The Jackson Laboratory, USA) with the floxed MR mice. $\mathrm{MRf} / \mathrm{f}$ littermates lacking the Cre transgene were used as controls.

Floxed MR mice were crossed with Tie2-Cre mice (strain: B6.Cg-Tg(Tek-cre) 12Flv/J, The Jackson Laboratory) to obtain EC MR-/- (MRflox/flox//Tie2-Cre) mice and corresponding MR $\mathrm{f} / \mathrm{f}$ littermates as controls.

Animals were kept in pathogen-free conditions with food, water and litter, and housed in a 12-h light/12-h dark cycle. Anesthesia was induced by intramuscular ketamine $40 \mathrm{mg} / \mathrm{kg}$ and xylazine $4 \mathrm{mg} / \mathrm{kg}$ in rats, and intraperitoneal injection of ketamine $50 \mathrm{mg} / \mathrm{kg}$ and xylazine10 $\mathrm{mg} / \mathrm{kg}$ in mice. Animals were sacrificed by carbon dioxide inhalation or cervical dislocation.

\section{Model of corneal neovascularization. Vecadh-MR-KO mice and their MRf/f} littermates were used for the generation of corneal neovascularization. Only the right eye of each animal was used. The corneal epithelium was entirely removed up to the limbus with a surgical microsponge imbibed with $70 \%$ alcohol. A suture (7-0 silk) was made to maintain the eyelids closed until day 3 . At day 10 , mice were perfused with $200 \mu \mathrm{l}$ FITC-dextran $(5 \mathrm{mg} / \mathrm{ml}$, molecular weight 2,000,000, SigmaAldrich FD2000S, St-Quentin Fallavier, France) before sacrifice and enucleation. Eyes were then fixed in $4 \%$ paraformaldehyde (PFA) for $2 \mathrm{~h}$ and washed in phosphate buffered saline (PBS). Corneal buttons were dissected and flat-mounted. FITC-dextran perfused corneal neovessels were observed with a fluorescence microscope (Olympus, Rungis, France). A mosaic of pictures and quantification were obtained using ImageJ. The area of neovascularization was expressed as a percentage of the total corneal area.

Laser-induced CNV. After anesthesia and dilation of the pupils, coverslips were positioned on the cornea as a contact glass. For rats, six or eight burns (six burns in rat eyes used for fluorescein angiography and CNV quantification; eight burns in rat eyes used for QPCR and western blot analyses) were performed 2 to 3 optic disc diameters away from the optic nerve with an Argon laser $(532 \mathrm{~nm})$ mounted on a slit lamp $(175 \mathrm{~mW}, 0.1 \mathrm{~s}$, and $50 \mu \mathrm{m})$. For mice, four laser burns were induced at the 3, 6, 9 and 12 o'clock positions around the optic disc $(250 \mathrm{~mW}, 0.05 \mathrm{~s}$, and $50 \mu \mathrm{m}$ ). Both eyes of animals received laser induction. The presence of a bubble witnessed the rupture of Bruch's membrane and confirmed a successful laser impact.

Treatments. Treatments were introduced in the rat model of CNV. After laser photocoagulation, rats were divided into eight treatment groups: (1) daily subcutaneous injection of spironolactone diluted in $90 \%$ olive oil and 10\% dimethyl sulfoxide (DMSO) $(25 \mathrm{mg} / \mathrm{kg} /$ day) until sacrifice; (2) daily subcutaneous injection of vehicle (olive oil + DMSO) until sacrifice; (3) intravitreous injection (IVT) of anti-rat VEGF (5 $\mu \mathrm{l}$ of $1.5 \mu \mathrm{g} / \mu \mathrm{l}, \mathrm{RnD}$ system, Lille, France) at day 0; (4) coadministration of subcutaneous spironolactone with anti-VEGF IVT; (5) IVT of spironolactone-loaded PLGA MSs at day $0(5 \mu \mathrm{l}$ of $2.2 \mu \mathrm{g} / \mu \mathrm{l})$; (6) IVT of nonloaded PLGA MSs (5 $\mu \mathrm{l}$ ) at day 0; (7) Oral eplerenone (INSPRA ${ }^{\oplus}, 200 \mathrm{mg} / \mathrm{kg} / \mathrm{day}$, $0.2 \%$ in chow) from day 0 until sacrifice; (8) Control normal chow from day 0 until sacrifice. Wild-type C57BL/6 mice were also treated with daily subcutaneous injection of spironolactone $(50 \mathrm{mg} / \mathrm{kg} /$ day $)$ from day 0 to sacrifice, compared to vehicle injections (olive oil + DMSO).

Fluorescein angiography (FA). FA was performed 14 days (in rats) or 10 days (in mice) after laser induction. After pupil dilatation, fluorescein $(0.2 \mathrm{ml}$ of $10 \%$ fluorescein in saline) was injected intravenously in the tail of rats, or intraperitoneal $(0.1 \mathrm{ml})$ in mice. Early and late phase angiograms were recorded at 1-3 and 5-7 min, respectively, after fluorescein injection. Simultaneously, infrared images were acquired to detect the site and effective presence of laser burn. For each laserinduced lesion, fluorescein leakage was graded qualitatively by evaluating the increase in size/intensity of dye between the early and late phases. Angiographic scores were established by two blinded observers according to the following criteria: grade 0 , no hyperfluorescence; grade 1 , slight hyperfluorescence with no increase in intensity nor in size; grade 2 , hyperfluorescence increasing in intensity but not in size; grade 3, hyperfluorescence increasing both in intensity and size; grade 4 , hyperfluorescence size increase more than 2-diameter of the initial laser burn.

RPE-choroid flat-mounts and CNV quantifications. Two days after FA examination (time necessary for fluorescein elimination), eyes were enucleated, fixed in 4\% PFA for $15 \mathrm{~min}$ at room temperature and sectioned at the limbus; the cornea and lens were discarded. The retina was separated from the RPE-choroid complex. Eight radial incisions were made on the RPE-choroid, which was then flat-mounted and post-fixed with acetone for $15 \mathrm{~min}$ at $-20^{\circ} \mathrm{C}$. After washing with $0.1 \%$ Triton x100 in PBS, FITC-GSL I-Isolectin B4 (FL-1201, 1:200, Vector, AbCys, Paris, France) was applied overnight at $-4{ }^{\circ} \mathrm{C}$. After washing with PBS, the RPE-choroid was flat-mounted and observed with a confocal microscope (Zeiss LSM710, Le Pecq, France). Images of the CNV were captured with a digital video camera coupled to a computer system. Bruch ruptures could be easily observed at each laser spot. Horizontal optical sections (at $1 \mu \mathrm{m}$ intervals) were obtained from the $\mathrm{CNV}$ surface. The deepest focal plane in which the surrounding choroidal vascular network connecting to the lesion could be identified was judged to be the floor of the CNV lesion. The area of CNV-related fluorescence on each horizontal section was measured using the ImageJ software. The summation of the entire fluorescent area on z-stack images from the top to the bottom of the CNV was used as an index for the $\mathrm{CNV}$ volume.

Mice were perfused with $200 \mu \mathrm{l}$ FITC-dextran (FD2000S, $5 \mathrm{mg} / \mathrm{ml}$, molecular weight 2,000,000, Sigma-Aldrich) before enucleation. After RPE-choroidal flatmounting, the FITC-dextran perfused CNV was examined and analyzed as previously described.

Immunofluorescence of RPE-choroid flat-mounts. Three days after laser induction (peak of macrophage infiltration), RPE-choroid flat-mounts were also prepared for immunofluorescence. A polyclonal rabbit anti-IBA1 antibody (019-19741, 1:400, Wako, Neuss, Germany) was applied overnight at $-4{ }^{\circ} \mathrm{C}$. After washing with $0.1 \%$ Triton X100/PBS, the flat-mounts were incubated with AlexaFluo $^{\oplus} 594$ goat anti-rabbit IgG (A-11012, 1:200, ThermoFisherScientific, Saint Aubin, France), the nuclei were counterstained with DAPI. Images of IBA1 positive macrophage/microglia were observed and captured with a confocal microscope. The IBA1 positive area was measured using the ImageJ software. The average fluorescent area per burn per eye was calculated.

Immunohistochemistry. Enucleated eyes were fixed in $4 \%$ PFA for $2 \mathrm{~h}$, dehydrated and embedded in paraffin. Eight- $\mu \mathrm{m}$-thick sections were deparaffinized in xylene, hydrated in a graded alcohol series, and washed in PBS-Tween (PBST). After antigen retrieval by heating in citrate buffer and inactivation of endogenous peroxidase by $3 \% \mathrm{H} 2 \mathrm{O} 2$, sections were incubated with $3 \%$ normal horse serum to reduce the non-specific signal. Mouse monoclonal anti-MR 6G1 (1:100, kindly provided by C. Gomez-Sanchez, Division of Endocrinology, University of Mississippi Medical Center, Jackson, MS) was applied overnight at $4^{\circ} \mathrm{C}$. After washing in PBST, sections were incubated with the biotinylated horse anti-mouse IgG BA2000 (1:250, Vector, AbCys, Paris, France) for $45 \mathrm{~min}$ at room temperature. Amplification of the signal was obtained with Tyramide Signal Amplification (TSA) kit (Perkin Elmer, Courtaboeuf, France) according to the manufacturer's instructions. Signal was revealed with 3,3'-diaminobenzidine tetrahydrochloride. Negative controls were performed without a primary antibody.

Quantitative PCR. For QPCR in rats, eight laser impacts per eye were performed In all these experiments, $n$ corresponds to the number of animals. Three days after laser induction, the rat neuroretinas and RPE-choroid-sclera complexes were carefully dissected from enucleated eyes, snap-frozen in liquid nitrogen and stored at $-80{ }^{\circ} \mathrm{C}$ until use. Total RNA was isolated from tissues using the RNeasyPlus Mini Kit (Qiagen, Courtaboeuf, France) according to the manufacturer's instructions. First-strand complementary DNA was synthesized from the total mRNA using random primers (ThermoFisher Scientific) and SuperScript II reverse transcriptase (ThermoFisher Scientific). Transcript levels of MR, MCP-1, IL6, IL1 $\beta$, TNFa, iNOS, VEGF-A, PIGF, HIF1a, ANGPTL4, TGF $\beta$, and DCN were analyzed by quantitative real-time PCR performed in 7500 Real-Time PCR System (Applied Biosystems, Foster City, CA, USA) with either SYBR Green or TaqMan detection. HPRT1 and 18S were used as housekeeping genes. Delta CT threshold calculation was used for relative quantification of results. Primers are as follows: MR, $5^{\prime}$-TAA GTTTCCCCACGTGGTTC-3' (forward), 5'-ATCCACGTCTCATGGCTTTC-3' (reverse); MCP-1, 5'-CCTGCTGCTACTCATTCAC-3' (forward), 5'-TCT CAC T TG GTT CTG GTC C-3' (reverse); VEGF-A, 5'-GCGGGCTGCTGCAATG-3' (forward), 5'-TGCAACGCGAGTCTGTGTTT-3' (reverse); PlGF, 5'-GTCCTTCT GAGTCGCTGTAG-3' (forward), 5'-TTCCTCCTTTCTGCCTTTGT-3' (reverse); HIF1a, 5'-GGTGGATATGTCTGGGTTGAG-3' (forward), 5'-TTCAACTGGTTT GAGGACAGA-3' (reverse); ANGPTL4, 5' ${ }^{\prime}$-TTCTCTACCTGGGACCAAGA-3' (forward), 5'-CTGTAGTGGATAGTAGCGGC-3' (reverse); TGF $\beta$, 5'-CAG AAG TTG GCA TGG TAG CC-3' (forward), 5'-TGC TTC AGC TCC ACA GAG AA-3 
(reverse); DCN, 5'-CTGCTATTCCTCAAGGTCTG-3' (forward), 5'-AGGAACAT TAGCCAGACTGC-3' (reverse); HPRT1, 5'-GCGAAAGTGGAAAAGCCAAGT3' (forward), 5'-GCCACATCAACAGGACTCTTGTAG-3' (reverse); 18S, 5'-TGC AATTATTCCCCATGAACG-3' (forward), 5'-GCTTATGACCCGCACTTACTG G-3' (reverse). IL6,IL1 $\beta$, TNFa, iNOS were assayed using Thermo Fisher TaqMan (Assays IDs Rn01410330_m1,Rn00676333_g1, Rn01525859_g1,Rn00561646_m1) with HPRT1 as housekeeping gene (Assay ID Rn01527840_m1).

IVT of recombinant DCN protein. We used recombinant mouse DCN protein (R\&D Systems) that shares $87 \%$ amino-acid sequence identity with rat DCN. IVT of DCN was performed after laser induction at a final concentration of $1 \mu \mathrm{g} / \mathrm{ml}$ or $10 \mu \mathrm{g} / \mathrm{ml}$ in the rat vitreous. FA was performed at day 14 , and CNV quantification on choroidal flat-mounts at day 16. Rat was chosen for this experiment as CNV is more reproducibly induced in rats than in mice, the eye size is larger allowing collection of more material and repeated injections are easier to perform in the larger rat eye.

DCN silencing by siRNA in CNV model. Rat DCN stealth siRNAs (set of 3, RSS307035, RSS307036, RSS307037) were purchased from ThermoFisher Scientific. Stock solution was made by adding $1 \mathrm{ml} \mathrm{H}_{2} \mathrm{O}$ to $20 \mathrm{nMol}$ DCN siRNA set. Before IVT, for each rat, siRNA injection solution was prepared by mixing $2 \mu$ l siRNA stock solution with $10 \mu \mathrm{l}$ Mirus transfection reagent and vortexed. After laser induction, $5 \mu \mathrm{l}$ of siRNA solution was injected in each eye of a rat. Control rat eyes were injected with scrambled siRNA (Stealth ${ }^{\text {tw }}$ RNAi negative control, medium GC duplex, ThermoFischer Scientific) prepared according to manufacturer's instruction. Animals were sacrificed at day 2 after laser induction, and the RPE-choroid complexes were retrieved for western blotting. To test the effect of DCN silencing on CNV formation, DCN siRNA was injected intravitreously in rat eyes at day 0 and day 3 after laser induction. FA was then performed at day 14 to evaluate the permeability of choroidal neovessels. CNV was stained using FITC-GSL I-Isolectin B4 on RPE-choroid flat-mounts and quantified using Image-J.

Western blotting. For protein analysis, we performed eight laser impacts per rat eye. The experiment was repeated twice independently. The dissected RPEchoroidal samples were processed using standard methods ${ }^{23}$ for western blotting. Briefly, equal amounts of protein $(20 \mu \mathrm{g})$ were separated on Novex ${ }^{\circledast} 4-20 \%$ TrisGlycine gel (Thermo Fisher Scientific), transferred to nitrocellulose, and the blots were incubated with rabbit anti-DCN (ab175404, 1:1000, Abcam, Cambridge, UK) at $4{ }^{\circ} \mathrm{C}$ overnight. The membranes were then washed, incubated with HRP goat anti-rabbit IgG antibody (PI-1000, 1:5000, vector) for $1 \mathrm{~h}$ at room temperature, and developed using ECL Plus western blotting detection reagents (GE healthcare, Orsay, France). Scans of blots were provided as Supplementary Fig. 10. The goat anti-Actin (sc1616, 1:2500, Santa Cruz Biotechnology, Heidelberg, Germany) was used as internal control.

Statistical analysis. In rat and mouse CNV models, in order to take into account simultaneously the correlation between the two eyes of an animal and the correlation for repeated measurements in the same eye (in case of repeated impacts), a linear mixed model for repeated measures LMMRM (known to be robust to the normality assumption) was used, including treatment (or mutation), side, time and treatment $\mathrm{x}$ time as fixed effects and animal as a random effect. No adjustment for multiplicity was used at this level, knowing the exploratory purpose of the preclinical data.

When pooling different experiments for comparison purposes, a specific model (LMMRM) on control animals only was performed to check the comparability and the lack of heterogeneity between experiments. As no significant difference was shown between controls (no experiment effect), the pooling was considered interpretable. NC.

Analyses were performed in SAS ${ }^{\circledast} 9.4$ for Windows, SAS Institute Inc., Cary,

For data related to QPCR and western blot, comparison between two groups was performed using Mann-Whitney $U$-test. Comparison between multiple groups was analyzed using non-parametric Kruskal-Wallis followed by Dunns test (GraphPad Prism 5 for Windows, GraphPad Software Inc., San Diego, CA, USA). $p$-values of 0.05 or less were considered significant.

RNA-sequencing and data analysis. Male 8-week-old Lewis rats were injected intravitreously with aldosterone ( $20 \mathrm{nM}$ final concentration in the vitreous) and sacrificed after $24 \mathrm{~h}$. Control rats received vehicle injection. The whole retinas (comprising neuroretina and RPE-choroid complex) were isolated for RNAsequencing. Total RNA was extracted using the RNeasyMini Kit (Qiagen) including DNase I treatment. RNA integrity was checked on the Agilent 2100 Bioanalyzer. At least three independent biological replicate samples were sequenced and used for downstream analysis. RNA-sequencing was performed on Illumina HiSeq2000 platform. The average number of reads per sample was $27 \mathrm{M}$. Reads from each sample were processed as follows. First, reads were trimmed using an in-house Perl script with a minimum phred quality of 20 per vase and a minimum read length of $30 \mathrm{bp}$. On average, $24 \%$ of reads per sample were discarded. The resulting reads were later aligned to the Rattus Norvegicus genome assembly 3.4 (from Ensembl) using Tophat version 2.0.10. At least 12 million reads were aligned to the genome for each sample. We next quantified gene expression to obtain read count and FPKM values. The non-adjusted read counts for each gene were used for statistical calculation of global differential expression using DESeq2. Differentially expressed genes were selected at an adjusted $p$-values of $\leq 0.05$ and fold changes $>1.5$.

Differential expression between aldosterone- and vehicle-injected rats was also analyzed using the GOlandscape, a threshold-free gene ontology tool (The GOlanscape R-package and a detailed description of the tool are available at the website https://github.com/andreaprunotto/GOlandscape). This software computes a stepwise $\mathrm{GO}$-analysis all over the range of $\mathrm{DE}$ significance, assigning a $p$-value for each GO term at each step. Then it selects the most significant annotated categories in the range, displaying them in combination with the most represented annotated genes within these terms. The output is a heatmap, which enables the analyst to identify quickly which gene is involved in which process (at the maximum level of statistical significance), independently on an arbitrary choice of the DE genes list.

Code availability. The $\mathrm{R}$ code of GOlandscape used in this study and related documentation are available at the websites https://github.com/andreaprunotto/ GOlandscape and https://zenodo.org/record/1474122

Reporting Summary. Further information on experimental design is available in the Nature Research Reporting Summary linked to this Article.

\section{Data availability}

All relevant data are available from the corresponding author upon reasonable request. RNA-sequencing data are available from ArrayExpress database at EMBLEBI under accession number E-MTAB-7438. The images from figures are available at figshare. A Reporting Summary for this Article is available as a Supplementary Information file.

Received: 30 March 2018 Accepted: 11 December 2018

Published online: 21 January 2019

\section{References}

1. Wong, W. L.et al. Global prevalence of age-related macular degeneration and disease burden projection for 2020 and 2040: a systematic review and metaanalysis. Lancet Glob. Health 2, e106-e116 (2014).

2. Mitchell, P., Liew, G., Gopinath, B. \& Wong, T. Y. Age-related macular degeneration. Lancet 392, 1147-1159 (2018).

3. Kaneko, H. et al. DICER1 deficit induces Alu RNA toxicity in age-related macular degeneration. Nature 471, 325-330 (2011).

4. Celkova, L. \& Doyle, S. L. \& Campbell, M. . NLRP3 inflammasome and pathobiology in AMD. J. Clin. Med. 4, 172-192 (2015).

5. Whitmore, S. S. et al. Complement activation and choriocapillaris loss in early AMD: implications for pathophysiology and therapy. Prog. Retin. Eye Res. $\mathbf{4 5}$, 1-29 (2015)

6. Witmer, A. N., Vrensen, G. F. J. M., Van Noorden, C. J. F. \& Schlingemann, R. $\mathrm{O}$. Vascular endothelial growth factors and angiogenesis in eye disease. Prog. Retin. Eye Res. 22, 1-29 (2003).

7. Bhutto, I. \& Lutty, G. Understanding age-related macular degeneration (AMD): relationships between the photoreceptor/retinal pigment epithelium/ Bruch's membrane/choriocapillaris complex. Mol. Asp. Med. 33, 295-317 (2012).

8. Campochiaro, P. A. Molecular pathogenesis of retinal and choroidal vascular diseases. Prog. Retin. Eye Res. 49, 67-81 (2015).

9. Zhang, S. X. \& Ma, J. Ocular neovascularization: Implication of endogenous angiogenic inhibitors and potential therapy. Prog. Retin. Eye Res. 26, 1-37 (2007).

10. Ba, J. et al. Intravitreal anti-VEGF injections for treating wet age-related macular degeneration: a systematic review and meta-analysis. Drug Des. Devel. Ther. 9, 5397-5405 (2015).

11. Rezar-Dreindl, S. et al. The intraocular cytokine profile and therapeutic response in persistent neovascular age-related macular degeneration. Invest. Ophthalmol. Vis. Sci. 57, 4144-4150 (2016).

12. Comparison of Age-related Macular Degeneration Treatments Trials (CATT) Research Group. et al. Five-year outcomes with anti-vascular endothelial growth factor treatment of neovascular age-related macular degeneration: The comparison of age-related macular degeneration treatments trials. Ophthalmology 123, 1751-1761 (2016).

13. Rush, R. B., Rush, S. W., Aragon, A. V. \& Ysasaga, J. E. Evaluation of choroidal neovascularization with indocyanine green angiography in neovascular agerelated macular degeneration subjects undergoing intravitreal bevacizumab therapy. Am. J. Ophthalmol. 158, 337-344 (2014). 
14. Framme, C., Panagakis, G. \& Birngruber, R. Effects on choroidal neovascularization after anti-VEGF Upload using intravitreal ranibizumab, as determined by spectral domain-optical coherence tomography. Invest. Ophthalmol. Vis. Sci. 51, 1671-1676 (2010).

15. Yang, S., Zhao, J. \& Sun, X. Resistance to anti-VEGF therapy in neovascular age-related macular degeneration: a comprehensive review. Drug Des. Devel. Ther. 10, 1857-1867 (2016).

16. Miller, J. W. Beyond VEGF-the weisenfeld lecture. Invest. Ophthalmol. Vis. Sci. 57, 6911-6918 (2016).

17. Arnold, J. J., Markey, C. M., Kurstjens, N. P. \& Guymer, R. H. The role of subretinal fluid in determining treatment outcomes in patients with neovascular age-related macular degeneration-a phase IV randomised clinical trial with ranibizumab: the FLUID study. BMC Ophthalmol. 16, 31 (2016).

18. CATT Research Group, et al. Ranibizumab and bevacizumab for neovascular age-related macular degeneration. N. Engl. J. Med. 364, 1897-1908 (2011).

19. Gewaily, D., Muthuswamy, K. \& Greenberg, P. B. Intravitreal steroids versus observation for macular edema secondary to central retinal vein occlusion. Cochrane Database Syst. Rev. CD007324 (2015). https://doi.org/10.1002/ 14651858.CD007324.pub3

20. Cao, J. H., Mulvahill, M., Zhang, L., Joondeph, B. C. \& Dacey, M. S. Dexamethasone intravitreal implant in the treatment of persistent uveitic macular edema in the absence of active inflammation. Ophthalmology 121, 1871-1876 (2014).

21. Whitcup, S. M., Cidlowski, J. A., Csaky, K. G. \& Ambati, J. Pharmacology of corticosteroids for diabetic macular edema. Invest. Ophthalmol. Vis. Sci. 59, $1-12$ (2018)

22. Zhao, M. et al. The neuroretina is a novel mineralocorticoid target: aldosterone up-regulates ion and water channels in Müller glial cells. Faseb J. 24, 3405-3415 (2010).

23. Zhao, M. et al. Mineralocorticoid receptor is involved in rat and human ocular chorioretinopathy. J. Clin. Invest. 122, 2672-2679 (2012).

24. Daruich, A. et al. Central serous chorioretinopathy: Recent findings and new physiopathology hypothesis. Prog. Retin. Eye Res. 48, 82-118 (2015).

25. Daruich, A. et al. Oral mineralocorticoid-receptor antagonists: Real-life experience in clinical subtypes of nonresolving central serous chorioretinopathy with chronic epitheliopathy. Transl. Vis. Sci. Technol. 5, 2 (2016).

26. Bousquet, E. et al. Spironolactone for nonresolving central serous chorioretinopathy: A randomized controlled crossover study. Retina 35 2505-2515 (2015).

27. Jaisser, F. \& Farman, N. Emerging roles of the mineralocorticoid receptor in pathology: Toward new paradigms in clinical pharmacology. Pharmacol. Rev. 68, 49-75 (2016).

28. Crum, R., Szabo, S. \& Folkman, J. A new class of steroids inhibits angiogenesis in the presence of heparin or a heparin fragment. Science 230, 1375-1378 (1985).

29. Fujii, M. et al. Aldosterone inhibits endothelial morphogenesis and angiogenesis through the downregulation of vascular endothelial growth factor receptor-2 expression subsequent to peroxisome proliferator-activated receptor gamma. J. Steroid Biochem. Mol. Biol. 129, 145-152 (2012).

30. Michel, F. et al. Aldosterone enhances ischemia-induced neovascularization through angiotensin II-dependent pathway. Circulation 109, 1933-1937 (2004).

31. Miternique-Grosse, A. et al. Antiangiogenic effects of spironolactone and other potassium-sparing diuretics in human umbilical vein endothelial cells and in fibrin gel chambers implanted in rats. J. Hypertens. 24, 2207-2213 (2006).

32. Wilkinson-Berka, J. L., Tan, G., Jaworski, K. \& Miller, A. G. Identification of a retinal aldosterone system and the protective effects of mineralocorticoid receptor antagonism on retinal vascular pathology. Circ. Res. 104, 124-133 (2009).

33. Grossniklaus, H. E., Kang, S. J. \& Berglin, L. Animal models of choroidal and retinal neovascularization. Prog. Retin. Eye Res. 29, 500-519 (2010).

34. Kolkhof, P. \& Bärfacker, L. 30 Years of the mineralocorticoid receptor: Mineralocorticoid receptor antagonists: 60 years of research and development. J. Endocrinol. 234, T125-T140 (2017).

35. Zhao, M. et al. Tolerance of high and low amounts of PLGA microspheres loaded with mineralocorticoid receptor antagonist in retinal target site. $J$. Control. Release 266, 187-197 (2017).

36. Schäfer, N. et al. Endothelial mineralocorticoid receptor activation mediates endothelial dysfunction in diet-induced obesity. Eur. Heart J. 34, 3515-3524 (2013).

37. Miller, J. W., Le Couter, J., Strauss, E. C. \& Ferrara, N. Vascular endothelial growth factor a in intraocular vascular disease. Ophthalmology 120, 106-114 (2013).

38. Olsen, T. W., Feng, X., Kasper, T. J., Rath, P. P. \& Steuer, E. R. Fluorescein angiographic lesion type frequency in neovascular age-related macular degeneration. Ophthalmology 111, 250-255 (2004).
39. Jung, J. J. et al. The incidence of neovascular subtypes in newly diagnosed neovascular age-related macular degeneration. Am. J. Ophthalmol. 158, 769-779.e2 (2014).

40. Heier, J. S. et al. Intravitreal aflibercept (VEGF trap-eye) in wet age-related macular degeneration. Ophthalmology 119, 2537-2548 (2012).

41. Hussain, R. M. \& Ciulla, T. A. Emerging vascular endothelial growth factor antagonists to treat neovascular age-related macular degeneration. Exp. Opin. Emerg. Drugs 22, 235-246 (2017).

42. Kapoor, K. G., Todi, N. \& Wagner, A. L. Mineralocorticoid antagonists as adjuncts in neovascular age-related macular degeneration. Ophthalmol. Ther. 6, 141-146 (2017).

43. Grunin, M., Hagbi-Levi, S. \& Chowers, I. The role of monocytes and macrophages in age-related macular degeneration. Adv. Exp. Med. Biol. 801, 199-205 (2014).

44. Combadière, C. et al. CX3CR1-dependent subretinal microglia cell accumulation is associated with cardinal features of age-related macular degeneration. J. Clin. Invest. 117, 2920-2928 (2007).

45. Usher, M. G. et al. Myeloid mineralocorticoid receptor controls macrophage polarization and cardiovascular hypertrophy and remodeling in mice. J. Clin. Invest. 120, 3350-3364 (2010).

46. Barrera-Chimal, J. et al. The myeloid mineralocorticoid receptor controls inflammatory and fibrotic responses after renal injury via macrophage interleukin-4 receptor signaling. Kidney Int. 93, 1344-1355 (2018).

47. Järveläinen, H., Sainio, A. \& Wight, T. N. Pivotal role for decorin in angiogenesis. Matrix Biol. 43, 15-26 (2015).

48. Frikeche, J., Maiti, G. \& Chakravarti, S. Small leucine-rich repeat proteoglycans in corneal inflammation and wound healing. Exp. Eye Res. 151, 142-149 (2016).

49. Keenan, T. D. L. et al. Mapping the differential distribution of proteoglycan core proteins in the adult human retina, choroid, and sclera. Invest. Ophthalmol. Vis. Sci. 53, 7528-7538 (2012).

50. Gubbiotti, M. A., Vallet, S. D., Ricard-Blum, S. \& Iozzo, R. V. Decorin interacting network: A comprehensive analysis of decorin-binding partners and their versatile functions. Matrix Biol. 55, 7-21 (2016).

51. Nelimarkka, L. et al. Decorin is produced by capillary endothelial cells in inflammation-associated angiogenesis. Am. J. Pathol. 158, 345-353 (2001).

52. Schönherr, E. et al. Decorin deficiency leads to impaired angiogenesis in injured mouse cornea. J. Vasc. Res. 41, 499-508 (2004).

53. Neill, T., Schaefer, L. \& Iozzo, R. V. Decorin as a multivalent therapeutic agent against cancer. Adv. Drug Deliv. Rev. 97, 174-185 (2016).

54. Neill, T. et al. Decorin antagonizes the angiogenic network: concurrent inhibition of Met, hypoxia inducible factor $1 \alpha$, vascular endothelial growth factor A, and induction of thrombospondin-1 and TIMP3. J. Biol. Chem. 287, 5492-5506 (2012)

55. Du, S., Wang, S., Wu, Q., Hu, J. \& Li, T. Decorin inhibits angiogenic potentia of choroid-retinal endothelial cells by downregulating hypoxia-induced Met, Rac1, HIF-1 $\alpha$ and VEGF expression in cocultured retinal pigment epithelial cells. Exp. Eye Res. 116, 151-160 (2013).

56. Xaus, J., Comalada, M., Cardó, M., Valledor, A. F. \& Celada, A. Decorin inhibits macrophage colony-stimulating factor proliferation of macrophages and enhances cell survival through induction of p27(Kip1) and p21(Waf1). Blood 98, 2124-2133 (2001)

57. Mohan, R. R. et al. Targeted decorin gene therapy delivered with adenoassociated virus effectively retards corneal neovascularization in vivo. PLoS ONE 6, e26432 (2011).

58. Wang, X. et al. TGF- $\beta$ participates choroid neovascularization through Smad2/3-VEGF/TNF- $\alpha$ signaling in mice with Laser-induced wet age-related macular degeneration. Sci. Rep. 7, 9672 (2017).

59. Yuan, X. et al. Quantitative proteomics: comparison of the macular Bruch membrane/choroid complex from age-related macular degeneration and normal eyes. Mol. Cell. Proteom. 9, 1031-1046 (2010).

60. Gergely, R. et al. Mineralocorticoid receptor antagonist treatment in bilateral chronic central serous chorioretinopathy: A comparative study of exudative and nonexudative fellow eyes. Retina 37, 1084-1091 (2017).

61. Funder, J. 30 years of the mineralocorticoid receptor: Mineralocorticoid receptor activation and specificity-conferring mechanisms: a brief history. $J$. Endocrinol. 234, T17-T21 (2017).

62. Barrera-Chimal, J. et al. Benefit of mineralocorticoid receptor antagonism in AKI: Role of vascular smooth muscle Racl. J. Am. Soc. Nephrol. 28, 1216-1226 (2017).

63. Clausen, B. E., Burkhardt, C., Reith, W., Renkawitz, R. \& Förster, I. Conditional gene targeting in macrophages and granulocytes using LysMcre mice. Transgenic. Res. 8, 265-277 (1999).

64. Mueller, K. B. et al. Endothelial mineralocorticoid receptors differentially contribute to coronary and mesenteric vascular function without modulating blood pressure. Hypertension 66, 988-997 (2015). 
65. Fraccarollo, D. et al. Deletion of cardiomyocyte mineralocorticoid receptor ameliorates adverse remodeling after myocardial infarction. Circulation 123, 400-408 (2011).

66. Wang, Y. et al. Ephrin-B2 controls VEGF-induced angiogenesis and lymphangiogenesis. Nature 465, 483-486 (2010).

67. Wendling, O., Bornert, J. -M., Chambon, P. \& Metzger, D. Efficient temporally-controlled targeted mutagenesis in smooth muscle cells of the adult mouse. Genesis 47, 14-18 (2009).

\section{Acknowledgements}

We thank INSERM, the Agence Nationale de la Recherche (ANR Mineraloret ANR-11BSV1-0022, and ROCK-SUR-MeR ANR-15-CE18-0032), the Fondation pour la Recherche Médicale (FRM Visual System 2013, DVS20131228894) and the Swiss National Science Foundation (grant \#156260 to C. R.) for financial support. This research was also supported by grants from MAT2013-43127- R and MAT2017-83858-C2-1 MINECO/AEI/FEDER,

UE. We thank Christophe Klein from Center d'Histologie, d'Imagerieet de Cytométrie, and Georges Zadigue from Center d'Explorations Fonctionnelles of Center de Recherche des Cordeliers for their technical support. We thank Maëva Dupuis-Deniaud, MDSTAT Consulting Lyon, France for her assistance in the statistical analysis.

\section{Author contributions}

M.Z. designed and performed the pre-clinical experiments, analyzed the data, wrote, revised and edited the manuscript. I.M. designed, performed, analyzed the clinical data and wrote and revised the manuscript. E.G., X.L., X.X., A.A., M.S., and R.L. performed experiments and analyzed the data. M.D. performed experiments and analyzed RNAseq data. A.P. did the computation analysis of the RNAseq data. C.A. designed experiments and analyzed RNAseq data. C.R. supervised the analysis of the RNAseq. J.C. designed and performed experiments, analyzed the data. M.C.N performed experiments. S.L. did transgenic mice breeding and performed experiments. N.F. designed experiments and revised the manuscript. I.B. and R.H. designed and analyzed the PLGA microspheres. F.J. supervised the research, designed experiments and revised the manuscript. F.B.C. supervised the research, designed experiments, analyzed the data, wrote, and revised the manuscript.

\section{Additional information}

Supplementary Information accompanies this paper at https://doi.org/10.1038/s41467018-08125-6.

Competing interests: M.Z. and F.B.-C. are listed as inventors in a patent application related to the "Methods and pharmaceutical compositions for the treatment of choroidal neovascularization” (WO2017064121A1, https://patents.google.com/ patent/WO2017064121A1/en). The remaining authors declare no competing interests.

Reprints and permission information is available online at http://npg.nature.com/ reprintsandpermissions/

Journal peer review information: Nature Communications thanks the anonymous reviewers for their contribution to the peer review of this work.

Publisher's note: Springer Nature remains neutral with regard to jurisdictional claims in published maps and institutional affiliations.

(c) (i) Open Access This article is licensed under a Creative Commons Attribution 4.0 International License, which permits use, sharing, adaptation, distribution and reproduction in any medium or format, as long as you give appropriate credit to the original author(s) and the source, provide a link to the Creative Commons license, and indicate if changes were made. The images or other third party material in this article are included in the article's Creative Commons license, unless indicated otherwise in a credit line to the material. If material is not included in the article's Creative Commons license and your intended use is not permitted by statutory regulation or exceeds the permitted use, you will need to obtain permission directly from the copyright holder. To view a copy of this license, visit http://creativecommons.org/ licenses/by/4.0/.

(C) The Author(s) 2019 